\title{
The Role of Statins on Helicobacter pylori Eradication: Results from the European Registry on the Management of H. pylori (Hp-EuReg)
}

\author{
María Caldas ${ }^{1}{ }^{\circledR}$, Ángeles Pérez-Aisa ${ }^{2}$, , Bojan Tepes $^{3}$, Alma Keco-Huerga ${ }^{4}$, Luis Bujanda ${ }^{5}$, \\ Alfredo J. Lucendo ${ }^{6} \mathbb{D}$, Luis Rodrigo ${ }^{7}{ }^{\mathbb{D}}$, Dino Vaira ${ }^{8}$, Luis Fernández-Salazar ${ }^{9}$, Jose M. Huguet ${ }^{10}$, \\ Jorge Pérez-Lasala ${ }^{11}$, Natasa Brglez Jurecic ${ }^{12}$, Galina Fadeenko ${ }^{13}$, Jesús Barrio ${ }^{14} \oplus$, Miguel Areia ${ }^{15} \oplus$, \\ Juan Ortuño ${ }^{16}$, Rinaldo Pellicano ${ }^{17}$, Marcis Leja ${ }^{18}$, Javier Molina-Infante ${ }^{19}$, Pavel Bogomolov ${ }^{20}$, \\ Sergey Alekseenko ${ }^{21}$, Manuel Domínguez-Cajal ${ }^{22}$, Judith Gómez-Camarero ${ }^{23}$, Vassiliki Ntouli ${ }^{24}$, \\ Samuel J. Martínez-Domínguez ${ }^{25}$, Rafael Ruiz-Zorrilla ${ }^{26}$, Oscar Núñez ${ }^{27}$, Aiman Silkanovna Sarsenbaeva ${ }^{28}$, \\ Pedro Almela ${ }^{29}$, Perminder Phull ${ }^{30}{ }^{10}$, Marta Espada ${ }^{1}$, Ignasi Puig ${ }^{31}$, Olga P. Nyssen ${ }^{1}{ }^{\circledR}$, Francis Mégraud ${ }^{32}$, \\ Colm O'Morain ${ }^{33}$, Javier P. Gisbert ${ }^{1, *}$ (1) and on behalf of the Hp-EuReg Investigators ${ }^{\dagger}$
}

check for

updates

Citation: Caldas, M.; Pérez-Aisa, Á.; Tepes, B.; Keco-Huerga, A.; Bujanda, L.; Lucendo, A.J.; Rodrigo, L.; Vaira, D.; Fernández-Salazar, L.; Huguet, J.M.; et al. The Role of Statins on Helicobacter pylori Eradication: Results from the European Registry on the Management of H. pylori (Hp-EuReg). Antibiotics 2021, 10, 965. https:// doi.org/10.3390/antibiotics10080965

Academic Editors: Mitsushige Sugimoto and Jordi Vila

Received: 28 June 2021

Accepted: 3 August 2021

Published: 11 August 2021

Publisher's Note: MDPI stays neutral with regard to jurisdictional claims in published maps and institutional affiliations.

Copyright: (c) 2021 by the authors. Licensee MDPI, Basel, Switzerland. This article is an open access article distributed under the terms and conditions of the Creative Commons Attribution (CC BY) license (https:// creativecommons.org/licenses/by/ $4.0 /)$.
1 Centro de Investigación Biomédica en Red de Enfermedades Hepáticas y Digestivas (CIBEREHD), Gastroenterology Unit, Instituto de Investigación Sanitaria Princesa (IIS-IP), Hospital Universitario de La Princesa, Universidad Autónoma de Madrid (UAM), 28006 Madrid, Spain; maria.caldas@salud.madrid.org (M.C.); martaespadasanchez@gmail.com (M.E.); opn.aegredcap@aegastro.es (O.P.N.)

2 Digestive Unit, Hospital Costa del Sol and Red de Investigación en Servicios de Salud en Enfermedades Crónicas (REDISSEC), 29651 Marbella, Spain; drapereza@hotmail.com

3 AM DC Rogaska, 3250 Rogaska Slatina, Slovenia; bojan.tepes@siol.net

4 Department of Gastroenterology, Hospital de Valme, 41014 Sevilla, Spain; almakh94@hotmail.com

5 Centro de Investigación Biomédica en Red de Enfermedades Hepáticas y Digestivas (CIBERehd), Department of Gastroenterology, Hospital Donostia/Instituto Biodonostia, Universidad del País Vasco (UPV/EHU), 20014 San Sebastián, Spain; luis.bujanda@osakidetza.net

6 Centro de Investigación Biomédica en Red de Enfermedades Hepáticas y Digestivas (CIBEREHD), Instituto de Investigación Sanitaria Princesa (IIS-IP), Department of Gastroenterology, Hospital General de Tomelloso, 13700 Ciudad Real, Spain; ajlucendo@sescam.jccm.es

7 Gastroenterology Unit, Hospital Universitario Central de Asturias, 33011 Oviedo, Spain; lrrodrigo@uniovi.es

8 S. Orsola Malpighi Hospital, 40138 Bologna, Italy; berardino.vaira@unibo.it

9 Digestive Service, Hospital Clínico Universitario de Valladolid, 47003 Valladolid, Spain; lfernandezsa@saludcastillayleon.es

10 Gastroenterology Unit, Consorcio Hospital General Universitario de Valencia, 46014 Valencia, Spain; huguet_jos@gva.es

11 Gastroenterology Unit, University Hospital of Torrejón, 28850 Madrid, Spain; jperez@torrejonsalud.com

12 Diagnosticni Center Bled d.o.o., 4260 Bled, Slovenia; natasa.brglez.jurecic@gmail.com

13 Digestive Ukrainian Academy of Medical Sciences, 01030 Kyiv, Ukraine; g.fadeenko@gmail.com

14 Gastroenterology Unit, Hospital Universitario Río Hortega, Gerencia Regional de Salud de Castilla y León (SACYL), 47012 Valladolid, Spain; jbarrio@saludcastillayleon.es

15 Portuguese Oncology Institute, 3000-075 Coimbra, Portugal; miguel.areia@ipocoimbra.min-saude.pt

16 Digestive Service, Hospital Universitari i Politècnic La Fe, 46026 Valencia, Spain; jortunoc@comv.es

17 Molinette Hospital, Città della Salute e della Scienza di Torino, 10126 Turin, Italy; rpellicano@cittadellasalute.to.it

18 Digestive Diseases Center, GASTRO, Institute of Clinical and Preventive Medicine and Faculty of Medicine, University of Latvia, LV-1079 Riga, Latvia; cei@latnet.lv

19 Department of Gastroenterology, Hospital San Pedro de Alcántara, 10003 Cáceres and Centro de Investigación Biomédica en Red de Enfermedades Hepáticas y Digestivas (CIBERehd), 28006 Madrid, Spain; javier.molinai@salud-juntaex.es

20 Universal Clinic Private Medical Center, 129110 Moscow, Russia; bpo73@list.ru

21 Far Eastern State Medical University, 680000 Khabarovsk, Russia; nauka@mail.fesmu.ru

22 Digestive Service, Hospital San Jorge, 22004 Huesca, Spain; mdominguezc@salud.aragon.es

23 Department of Gastroenterology and Hepatology, Complejo Asistencial Universitario de Burgos, 09006 Burgos, Spain; jgomezcam@saludcastillayleon.es

24 General Hospital Pireaus, 18536 Pireaus, Greece; vassiliki.ntouli@gmail.com 
25 Digestive Service, Hospital Clínico Universitario Lozano Blesa and CIBERehd, 50009 Zaragoza, Spain; martinezdominguezsamuel@gmail.com

26 Digestive Service, Hospital de Sierrallana Torrelavega, 39300 Cantabria, Spain; rafael.ruiz@scsalud.es

27 Digestive Service, Hospital Universitario Sanitas La Moraleja, 28050 Madrid, Spain; onumar@gmail.com

28 Gastroenterologist Department of Regional Clinical Hospital №3, 454076 Chelyabinsk, Russia; aiman-ss@yandex.ru

29 Digestive Service, Hospital Universitari General de Castelló, 12004 Castellón, Spain; almela_ped@gva.es

30 Aberdeen Royal Infirmary, Aberdeen AB25 2ZN, UK; p.s.phull@abdn.ac.uk

31 Digestive Service, Althaia Xarxa Assistencial Universitària de Manresa and Universitat de Vic-Universitat Central de Catalunya (UVicUCC), 08242 Manresa, Spain; ipuig@althaia.cat

32 Laboratoire de Bactériologie, Hôpital Pellegrin, Bordeaux \& INSERM U1053 BaRITOn, Université de Bordeaux, 33076 Bordeaux, France; francis.megraud@chu-bordeaux.fr

33 Department of Clinical Medicine, Trinity College Dublin, D24 NR0A Dublin, Ireland; colmOMorain@rcpi.ie

* Correspondence: javier.p.gisbert@gmail.com; Tel.: +34-913-093-911; Fax: 34-915-204-013

+ The remaining list of authors, their affiliations, contributions and conflicts of interests are listed in online Supplementary File S1.

\begin{abstract}
Statins could increase the effectiveness of Helicobacter pylori eradication therapies due to their anti-inflammatory effect. The aim of this study was to analyze the impact of this therapeutic association in real life. This is a multicenter, prospective, non-interventional study aimed at evaluating the management of $H$. pylori by European gastroenterologists. Patients were registered in an e-CRF by AEG-REDCap from 2013 to 2020. The association between statin use and H. pylori eradication effectiveness was evaluated through multivariate analysis. Overall, 9988 and 705 patients received empirical and culture-guided treatment, respectively. Overall, statin use was associated with higher effectiveness in the empirical group (OR $=1.3 ; 95 \% \mathrm{CI}=1.1-1.5)$, but no association was found with first-line treatment effectiveness $(\mathrm{N}=7738)$; as an exception, statin use was specifically associated with lower effectiveness of standard triple therapy $(\mathrm{OR}=0.76 ; 95 \% \mathrm{CI}=0.59-0.99)$. In the rescue therapy empirical group $(\mathrm{N}=2228)$, statins were associated with higher overall effectiveness $(\mathrm{OR}=1.9$; $95 \% \mathrm{CI}=1.4-2.6)$. However, sub-analyses by treatment schemes only confirmed this association for the single-capsule bismuth quadruple therapy $(\mathrm{OR}=2.8 ; 95 \% \mathrm{CI}=1.3-5.7)$. No consistent association was found between statin use and $H$. pylori therapy effectiveness. Therefore, the addition of statins to the usual H. pylori treatment cannot be currently recommended to improve cure rates.
\end{abstract}

Keywords: statins; Helicobacter pylori; treatment

\title{
1. Introduction
}

Helicobacter pylori (H. pylori) is a Gram-negative bacterium involved in the etiopathogenesis of several common gastric diseases such as peptic ulcer, chronic gastritis, or gastric cancer, but also several extra-gastric diseases (e.g., iron deficiency anemia, idiopathic thrombocytopenic purpura, and vitamin B12 deficiency), and the list of possible associations is constantly increasing [1-3].

Despite numerous attempts, an effective, unique, and global eradication therapy (providing $\geq 90 \%$ success) has not been found, mainly due to the geographical variability of antibiotic resistance rates and their increase worldwide [1,4,5].

Several strategies have been proposed to optimize the success rate of eradication therapies, most of them focused on extending treatment duration, using more potent drugs to decrease gastric acidity (such as high doses of proton pump inhibitors (PPIs)), or using quadruple instead of triple therapies. Other strategies, such as the use of dual therapy combining PPI and amoxicillin administered four times daily for 14 days, have provided good results in the Asiatic setting but need to be evaluated specifically in the European context [5-11].

One strategy suggested to increase eradication effectiveness has been the addition of statins. Statins mainly inhibit the activity of 3-hydroxy-3-methylglutaryl coenzyme A 
(HGM-CoA) reductase, blocking the first step of the L-mevalonate pathway and reducing the production of cholesterol in hepatocytes and other tissues. This causes a drop in LDL-cholesterol levels, explaining the main role of these drugs in the prevention of cardiovascular diseases $[12,13]$. However, by inhibiting this step, other metabolites derived from the cholesterol synthesis cascade such as non-steroidal isoprenoid compounds (named farnesyl pyrophosphate (FPP) and geranylgeranyl pyrophosphate (GGPP)) are affected. These molecules play a role in the post-translational lipid modification of several proteins involved in guanosine triphosphatase (GTPase) activation and intracellular signaling. This is known as the "pleiotropic effect" of statins, which does not seem to be proportional to the magnitude of the decline in LDL-cholesterol levels [14-16].

This pleiotropic effect has been associated with anti-inflammatory properties involving infectious and autoimmune diseases or even neoplastic conditions, although definitive conclusions have not been obtained [17-23]. In addition, a hypothetical role in the healing of gastric inflammation has also been suggested [24,25], and some authors have also proposed a possible effect increasing the effectiveness of the traditional eradication therapies against H. pylori infection [26]. Little is known about the exact mechanism of action of these drugs on $\mathrm{H}$. pylori infection. Some authors have suggested that the reduction in cellular cholesterol caused by the use of statins could result in reduced VacA activity and attenuated CagAinduced inflammation in gastric cells and in the induction of autophagy in macrophages infected with $H$. pylori $[27,28]$. However, in a previously published study performed in mice, $H$. pylori viability was not reduced by pravastatin, even though a drop in inflammation in gastritis could be observed [24].

Considering the limited evidence in this field and the current necessity to optimize eradication therapies, we evaluated a cohort of patients from the long-term prospective clinical practice European Registry on the Management of H. pylori (Hp-EuReg) [29], with the aim of obtaining updated information on the role of statins on $\mathrm{H}$. pylori eradication.

\section{Results}

From May 2013 to January 2020, 10,915 cases participating in the Hp-EuReg were selected for analysis, based on whether they were or were not daily statin receivers as part of a chronic therapy prescribed for cardiovascular risk prevention. Quality criteria were applied, leading to a final number of 10,693 patients included for analysis (Figure 1). These patients came from 123 European hospitals from a total of 25 different countries (online Supplementary Table S1).

Patients were divided in two groups according to statin use: 2635 cases received statins during the $H$. pylori eradication treatment and 8058 patients were not statin-consumers. Both cohorts were heterogeneous concerning the following variables: gender (female vs. male), presence of allergy to penicillin (yes vs. no), indication for $H$. pylori diagnosis (no ulcer vs. ulcer disease), use of different PPI doses (low vs. standard vs. high), and length of eradication treatment (7 vs. 10 vs. 14 days) (Table 1). Other reported basal characteristics were similar in both cohorts: line of eradication treatment prescribed (first vs. rescue attempts), type of prescription administered (empirical vs. culture-guided), treatment compliance ( $<90 \%$ vs. $\geq 90 \%$ ), and presence vs. absence of adverse events (AEs) (Table 1 ).

The type of statin was reported in $13 \%$ of all statins consumers and were mainly: simvastatin $(n=155,45 \%)$, atorvastatin $(n=134,39 \%)$, rosuvastatin $(n=37,11 \%)$, and a last group named "others" $(n=17,5 \%)$ including other statins (e.g., pravastatin, pitavastatin, lovastatin, or fluvastatin) (Table 2). Statin dosages were not reported and therefore no further analysis could be performed based on this variable. The distribution of the eradication therapies administered according to the statin used is shown in online Supplementary Table S2. 


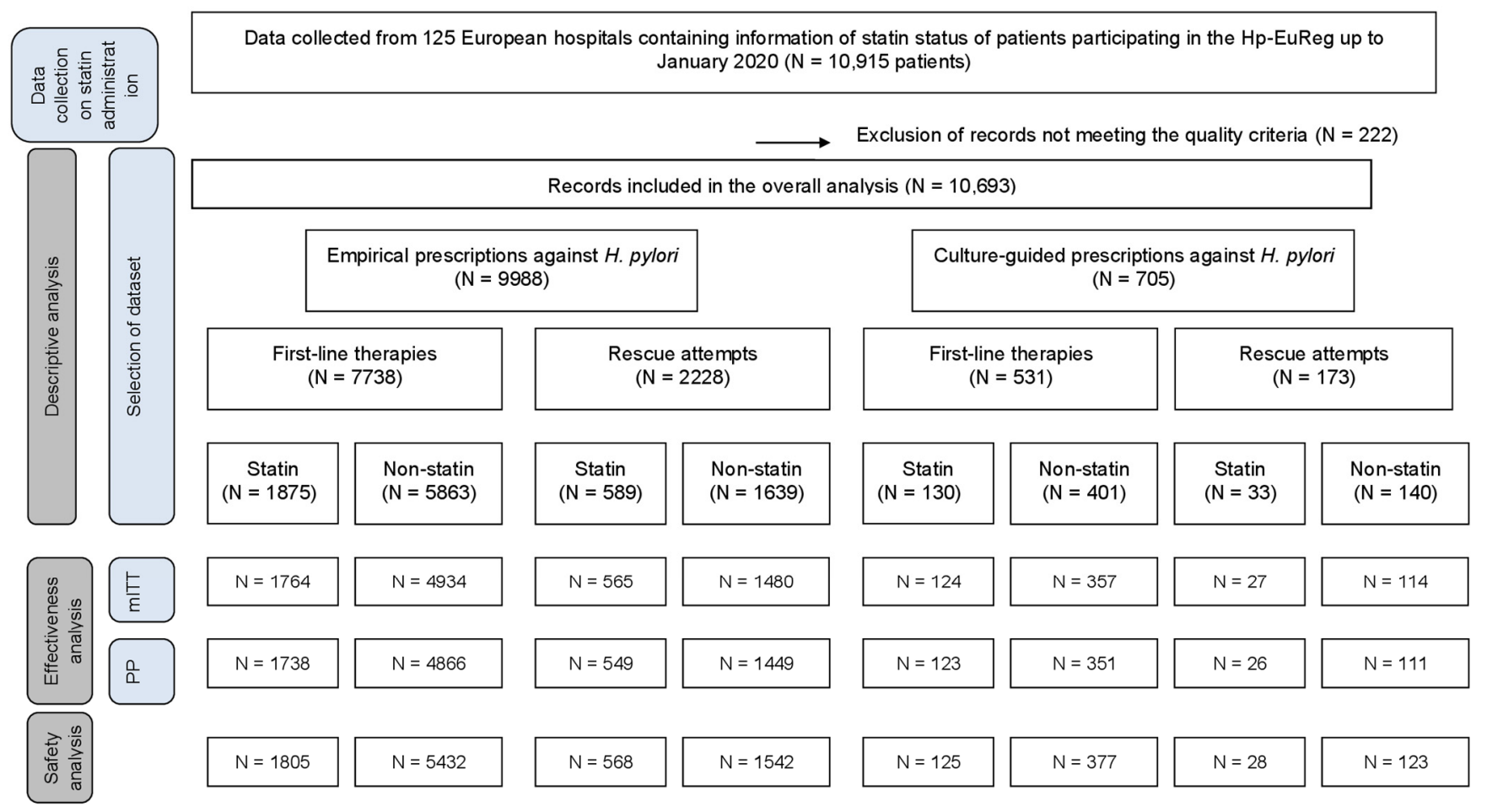

Figure 1. Flowchart of the patients included. $\mathrm{N}=$ number of patients treated. $\mathrm{mITT}=$ modified intention-to-treat analysis. $\mathrm{PP}=$ per protocol analysis.

Table 1. Demographic analysis and use of statins.

\begin{tabular}{|c|c|c|c|c|}
\hline & & $\begin{array}{c}\text { Statin Users, } \\
\text { N (\%) }\end{array}$ & $\begin{array}{l}\text { Non-Statin } \\
\text { Users, } \mathbf{N}(\%)\end{array}$ & $p$-Value \\
\hline Patients evaluated & & $2635(25)$ & $8058(75)$ & \\
\hline $\begin{array}{l}\text { Age (years) } \\
+/-S D\end{array}$ & & $63 \pm 10$ & $53 \pm 15$ & $<0.001$ \\
\hline Gender & $\begin{array}{c}\text { Females } \\
\text { Males }\end{array}$ & $\begin{array}{l}1518(58) \\
1114(42)\end{array}$ & $\begin{array}{l}5093(63) \\
2962(37)\end{array}$ & $<0.001$ \\
\hline Penicillin allergy & $\begin{array}{l}\text { Yes } \\
\text { No }\end{array}$ & $\begin{array}{c}141(5) \\
2494(95)\end{array}$ & $\begin{array}{c}347(4) \\
7711(96)\end{array}$ & 0.026 \\
\hline Indication & $\begin{array}{c}\text { No ulcer } \\
\text { Ulcer disease }\end{array}$ & $\begin{array}{c}1982(75.3) \\
649(25)\end{array}$ & $\begin{array}{l}6526(81) \\
1511(19)\end{array}$ & $<0.001$ \\
\hline Line of eradication & $\begin{array}{c}\text { First line } \\
\text { Rescue lines }\end{array}$ & $\begin{array}{c}2005(76) \\
622(24)\end{array}$ & $\begin{array}{l}6264(78) \\
1779(22)\end{array}$ & 0.097 \\
\hline Prescription & $\begin{array}{c}\text { Empirical } \\
\text { Culture-guided }\end{array}$ & $\begin{array}{c}2472(94) \\
163(6)\end{array}$ & $\begin{array}{c}7516(93) \\
542(7)\end{array}$ & 0.332 \\
\hline PPI dose & $\begin{array}{c}\text { Low } \\
\text { Standard } \\
\text { High }\end{array}$ & $\begin{array}{c}949(37) \\
626(24) \\
1006(39)\end{array}$ & $\begin{array}{l}3220(41) \\
1742(22) \\
2914(37)\end{array}$ & 0.002 \\
\hline Length (days) & $\begin{array}{c}7 \\
10 \\
14\end{array}$ & $\begin{array}{c}219(9) \\
1425(55) \\
929(36)\end{array}$ & $\begin{array}{c}941(12) \\
4195(53) \\
2719(35)\end{array}$ & 0.002 \\
\hline
\end{tabular}


Table 1. Cont.

\begin{tabular}{|c|c|c|c|c|}
\hline & & $\begin{array}{c}\text { Statin Users, } \\
\text { N (\%) }\end{array}$ & $\begin{array}{l}\text { Non-Statin } \\
\text { Users, N (\%) }\end{array}$ & $p$-Value \\
\hline \multirow{2}{*}{$\begin{array}{c}\text { Compliance } \\
(\geq 90 \%)\end{array}$} & Yes & $2476(98)$ & $7262(98)$ & \multirow{2}{*}{0.502} \\
\hline & No & $54(2)$ & $176(2)$ & \\
\hline \multirow{2}{*}{ Adverse events } & Yes & $594(24)$ & $1703(23)$ & \multirow{2}{*}{0.460} \\
\hline & No & $1934(76)$ & $5772(77)$ & \\
\hline \multirow{2}{*}{ Effectiveness } & mITT & $2190(88)$ & $5874(85)$ & $<0.001$ \\
\hline & PP & $2160(89)$ & $5818(86)$ & 0.001 \\
\hline
\end{tabular}

$\mathrm{N}=$ number of patients included. $\%$ = proportion of patients included. SD = standard deviation. Low dose PPI $=20 \mathrm{mg}$ omeprazole equivalents, two times per day, standard dose PPI $=40 \mathrm{mg}$ omeprazole equivalents, two times per day, high dose PPI $=60 \mathrm{mg}$ omeprazole equivalents, two times per day. $\mathrm{mITT}=$ modified-intentionto-treat effectiveness. PP = per protocol effectiveness. Significant $p$-values are highlighted in bold.

Table 2. Demographic analysis of the statin cohort stratified by type of statin.

\begin{tabular}{|c|c|c|c|c|c|c|}
\hline & & $\begin{array}{c}\text { Simvastatin } \\
\text { N (\%) }\end{array}$ & $\begin{array}{l}\text { Atorvastatin } \\
\qquad \mathbf{N}(\%)\end{array}$ & $\begin{array}{c}\text { Rosuvastatin } \\
\text { N (\%) }\end{array}$ & $\begin{array}{l}\text { Other } \\
\text { N (\%) }\end{array}$ & $p$-Value \\
\hline $\begin{array}{c}\text { Patients } \\
\text { evaluated }\end{array}$ & & $155(45)$ & $134(39)$ & $37(11)$ & $17(5)$ & \\
\hline $\begin{array}{l}\text { Age (years) } \\
+/-S D\end{array}$ & & $63 \pm 10$ & $63 \pm 10$ & $66 \pm 8$ & $63 \pm 13$ & 0.492 \\
\hline Gender & $\begin{array}{c}\text { Females } \\
\text { Males }\end{array}$ & $\begin{array}{l}86(55.5) \\
69(44.5)\end{array}$ & $\begin{array}{l}69(51.5) \\
65(48.5)\end{array}$ & $\begin{array}{l}19(51) \\
18(49)\end{array}$ & $\begin{array}{l}10(59) \\
7(41)\end{array}$ & 0.869 \\
\hline Penicillin allergy & $\begin{array}{l}\text { Yes } \\
\text { No }\end{array}$ & $\begin{array}{c}7(4.5) \\
148(95.5)\end{array}$ & $\begin{array}{c}4(3) \\
130(97)\end{array}$ & $\begin{array}{c}0(0) \\
37(100)\end{array}$ & $\begin{array}{c}1(6) \\
16(94)\end{array}$ & 0.532 \\
\hline Indication & $\begin{array}{l}\text { No ulcer } \\
\text { Ulcer disease }\end{array}$ & $\begin{array}{c}119(77) \\
35(23)\end{array}$ & $\begin{array}{l}91(68) \\
43(32)\end{array}$ & $\begin{array}{c}32(86.5) \\
5(13.5)\end{array}$ & $\begin{array}{l}14(82) \\
3(18)\end{array}$ & 0.070 \\
\hline $\begin{array}{l}\text { Treatment } \\
\text { attempt }\end{array}$ & $\begin{array}{c}\text { First } \\
\text { Rescue }\end{array}$ & $\begin{array}{c}120(77) \\
35(23)\end{array}$ & $\begin{array}{l}107(81) \\
25(19)\end{array}$ & $\begin{array}{l}30(81) \\
7(19)\end{array}$ & $\begin{array}{l}14(82) \\
3(18)\end{array}$ & 0.862 \\
\hline Prescription & $\begin{array}{c}\text { Empirical } \\
\text { Culture-guided }\end{array}$ & $\begin{array}{l}135(87) \\
20(13)\end{array}$ & $\begin{array}{l}108(81) \\
26(19)\end{array}$ & $\begin{array}{c}35(95) \\
2(5)\end{array}$ & $\begin{array}{l}15(88) \\
2(12)\end{array}$ & 0.142 \\
\hline PPI dose & $\begin{array}{l}\text { Low } \\
\text { Standard } \\
\text { High }\end{array}$ & $\begin{array}{l}75(49) \\
43(28) \\
36(23)\end{array}$ & $\begin{array}{l}80(62) \\
23(18) \\
26(20)\end{array}$ & $\begin{array}{c}29(78) \\
2(5) \\
6(16)\end{array}$ & $\begin{array}{l}10(59) \\
4(23) \\
3(18)\end{array}$ & 0.026 \\
\hline Length (days) & $\begin{array}{c}7 \\
10 \\
14\end{array}$ & $\begin{array}{c}17(11) \\
107(70) \\
28(18)\end{array}$ & $\begin{array}{l}17(13) \\
90(69) \\
23(18)\end{array}$ & $\begin{array}{c}5(13) \\
31(84) \\
1(3)\end{array}$ & $\begin{array}{c}2(12) \\
11(65) \\
4(23)\end{array}$ & 0.278 \\
\hline $\begin{array}{c}\text { Compliance } \\
(\geq 90 \%)\end{array}$ & $\begin{array}{l}\text { Yes } \\
\text { No }\end{array}$ & $\begin{array}{c}148(98) \\
3(2)\end{array}$ & $\begin{array}{c}128(98.5) \\
2(1.5)\end{array}$ & $\begin{array}{c}37(100) \\
0(0)\end{array}$ & $\begin{array}{c}16(100) \\
0(0)\end{array}$ & 0.789 \\
\hline Adverse events & $\begin{array}{l}\text { Yes } \\
\text { No }\end{array}$ & $\begin{array}{c}18(12) \\
136(88)\end{array}$ & $\begin{array}{c}21(16) \\
109(84)\end{array}$ & $\begin{array}{c}5(13.5) \\
32(86.5)\end{array}$ & $\begin{array}{c}2(12.5) \\
14(87.5)\end{array}$ & 0.751 \\
\hline Effectiveness & $\begin{array}{l}\text { mITT } \\
\text { PP }\end{array}$ & $\begin{array}{l}122(84) \\
122(85)\end{array}$ & $\begin{array}{l}112(91) \\
111(92)\end{array}$ & $\begin{array}{l}29(81) \\
29(81)\end{array}$ & $\begin{array}{l}16(100) \\
16(100)\end{array}$ & $\begin{array}{l}0.086 \\
0.074\end{array}$ \\
\hline
\end{tabular}

$\mathrm{N}=$ number of patients included. $\%$ = proportion of patients included. Others = includes pravastatin, pitavastatin, lovastatin and fluvastatin. $\mathrm{SD}=$ standard deviation. Low dose PPI $=20 \mathrm{mg}$ omeprazole equivalents, two times per day, standard dose PPI $=40 \mathrm{mg}$ omeprazole equivalents, two times per day, high dose PPI $=60 \mathrm{mg}$ omeprazole equivalents, two times per day. $\mathrm{mITT}=$ modified-intention-to-treat effectiveness. PP $=$ per protocol effectiveness. Significant $p$-values are highlighted in bold.

\subsection{Empirical Prescription}

Overall, $93 \%$ of the patients received an empirical prescription to treat $H$. pylori $(n=9988)$. Higher eradication rates were reported in the statin cohort than in non-statin users $(88 \%$ vs. $85 \% ; p<0.001$ in the univariate analysis, and an OR $=1.27 ; 95 \%$ CI: $1.1-1.5$; $p<0.05$ in the multivariate analysis). Other variables included in the multivariate analysis 
were also shown to be associated with an increase in mITT effectiveness, including good compliance, prescription of a first eradication attempt instead of a rescue regimen, male gender, presence of gastric ulcer disease, 10 and 14-day treatments (instead of 7-day treatment), and use of standard or high PPI doses (instead of low doses) (Tables 3 and 4).

Table 3. Univariate analysis of effectiveness and safety according to the type of prescription (empirical versus culture-guided).

\begin{tabular}{|c|c|c|c|c|c|c|c|c|c|c|}
\hline & \multirow[t]{2}{*}{ N (\%) } & \multicolumn{3}{|c|}{ mITT Effectiveness } & \multicolumn{3}{|c|}{ PP Effectiveness } & \multicolumn{3}{|c|}{ Adverse Events } \\
\hline & & N Total (\%) & $95 \% \mathrm{CI}$ & $p$-Value & N Total (\%) & $95 \% \mathrm{CI}$ & $p$-Value & N Total (\%) & $95 \%$ CI & $p$-Value \\
\hline \multicolumn{11}{|c|}{ Overall empirical therapies } \\
\hline $\begin{array}{l}\text { Statins } \\
\text { No S }\end{array}$ & $\begin{array}{l}2472(25) \\
7516(75)\end{array}$ & $\begin{array}{l}2330(88) \\
6414(85)\end{array}$ & $\begin{array}{l}87-89 \\
84-86\end{array}$ & $<0.001$ & $\begin{array}{c}2288(88.5) \\
6315(86)\end{array}$ & $\begin{array}{l}87-90 \\
85-87\end{array}$ & 0.001 & $\begin{array}{l}2375(24) \\
6975(23)\end{array}$ & $\begin{array}{l}22-25 \\
22-24\end{array}$ & 0.542 \\
\hline \multicolumn{11}{|c|}{ Overall culture-guided therapies } \\
\hline $\begin{array}{c}\text { Statins } \\
\text { No S }\end{array}$ & $\begin{array}{l}163(23) \\
542(77)\end{array}$ & $\begin{array}{l}151(90) \\
471(87)\end{array}$ & $\begin{array}{l}84-94 \\
83-90\end{array}$ & 0.266 & $\begin{array}{l}149(91) \\
462(88)\end{array}$ & $\begin{array}{l}85-95 \\
84-91\end{array}$ & 0.330 & $\begin{array}{l}153(22) \\
500(20)\end{array}$ & $\begin{array}{l}16-30 \\
17-24\end{array}$ & 0.589 \\
\hline
\end{tabular}

$\mathrm{N}=$ number of patients included. $\%=$ percentage of patients included. mITT = modified intention-to-treat. PP $=$ per protocol. $95 \% \mathrm{CI}=95 \%$ confidence interval. $\mathrm{S}=$ statins. Significant $p$-values are highlighted in bold.

Table 4. Multivariate analysis according to mITT effectiveness in empirical versus culture-guided prescriptions.

\begin{tabular}{|c|c|c|c|c|c|c|c|}
\hline & & \multicolumn{3}{|c|}{$\begin{array}{l}\text { Empirical Prescription } \\
\qquad \mathrm{N}=9988\end{array}$} & \multicolumn{3}{|c|}{$\begin{array}{l}\text { Culture-Guided Prescription } \\
\qquad N=705\end{array}$} \\
\hline & & OR & $95 \% \mathrm{CI}$ & $p$-Value & OR & $95 \% \mathrm{CI}$ & $p$-Value \\
\hline Gender & $\begin{array}{l}\text { Female } \\
\text { Male }\end{array}$ & $\begin{array}{c}1 \\
1.218\end{array}$ & $1.1-1.4$ & 0.004 & & NS & \\
\hline Indication & $\begin{array}{l}\text { No ulcer } \\
\text { Ulcer disease }\end{array}$ & $\begin{array}{c}1 \\
1.272\end{array}$ & $1.1-1.5$ & 0.005 & & NS & \\
\hline Treatment attempt & $\begin{array}{c}\text { First } \\
\text { Rescue }\end{array}$ & $\begin{array}{c}1 \\
0.522\end{array}$ & $0.45-0.60$ & $<0.001$ & $\begin{array}{c}1 \\
0.480\end{array}$ & $0.29-0.81$ & 0.006 \\
\hline Length (days) & $\begin{array}{c}7 \\
10 \\
14\end{array}$ & $\begin{array}{c}1 \\
1.375 \\
1.424\end{array}$ & $\begin{array}{l}1.1-1.7 \\
1.1-1.8\end{array}$ & $\begin{array}{l}0.001 \\
0.002\end{array}$ & & NS & \\
\hline PPI dose & $\begin{array}{c}\text { Low } \\
\text { Standard } \\
\text { High }\end{array}$ & $\begin{array}{c}1 \\
1.492 \\
2.026\end{array}$ & $\begin{array}{l}1.3-1.8 \\
1.7-2.4\end{array}$ & $\begin{array}{l}<0.001 \\
<0.001\end{array}$ & & NS & \\
\hline Compliance & $\begin{array}{l}\text { No } \\
\text { Yes }\end{array}$ & $\begin{array}{c}1 \\
5.224\end{array}$ & $3.6-7.6$ & $<0.001$ & $\begin{array}{c}1 \\
12.716\end{array}$ & $3.6-45$ & $<0.001$ \\
\hline Statin use & $\begin{array}{l}\text { No } \\
\text { Yes }\end{array}$ & $\begin{array}{c}1 \\
1.269\end{array}$ & $1.1-1.5$ & 0.002 & & NS & \\
\hline
\end{tabular}

$\mathrm{mITT}=$ modified intention-to-treat. $\mathrm{OR}=$ odds ratio. $95 \% \mathrm{CI}=95 \%$ confidence interval. $\mathrm{PPI}=$ proton pump inhibitor. Low dose PPI $=20 \mathrm{mg}$ omeprazole equivalents, two times per day. Standard dose PPI $=40 \mathrm{mg}$ omeprazole equivalents, two times per day. High dose PPI $=60 \mathrm{mg}$ omeprazole equivalents, two times per day. NS = statistically not significant.

No differences were found in the incidence rates of AEs: $24 \%$ vs. $23 \%$ in statin users and non-users, respectively.

Sub-analysis by treatment scheme in first and rescue therapy is described below.

\subsubsection{First-Line Therapy}

A total of 7738 patients received an empirical first-line therapy, of which 1875 patients were daily statin users and 5863 were non-users. No difference was found in terms of overall effectiveness, according to statin use, which was $88.5 \%$ in statin users and $87 \%$ in non-users (Table 5). The multivariate analysis showed no association between statin status and mITT effectiveness $(\mathrm{OR}=1.1 ; 95 \% \mathrm{CI}$ : $0.9-1.3 ; p=0.286)$. The variables that showed significant results in this context are shown in Table 6. 
Table 5. Effectiveness and safety in first-line empirically prescribed therapies.

\begin{tabular}{|c|c|c|c|c|c|c|c|c|c|c|c|}
\hline & & \multirow[t]{2}{*}{ N (R) } & \multicolumn{3}{|c|}{ mITT Effectiveness } & \multicolumn{3}{|c|}{ PP Effectiveness } & \multicolumn{3}{|c|}{ Adverse Events } \\
\hline & & & N (\%) & $95 \% \mathrm{CI}$ & $p$-Value & $\begin{array}{c}\text { N Total } \\
(\%)\end{array}$ & $95 \%$ CI & $p$-Value & N Total (\%) & $95 \%$ CI & $p$-Value \\
\hline \multirow{2}{*}{ Overall } & Statins & 1875 & $1764(88.5)$ & $87-90$ & \multirow{2}{*}{0.254} & $1738(89)$ & $87-90$ & \multirow{2}{*}{0.388} & $1805(20)$ & $18-22$ & \multirow{2}{*}{0.197} \\
\hline & No S & 5863 & $4934(87)$ & $86-88$ & & $4866(88)$ & $87-89$ & & $5432(21)$ & $20-23$ & \\
\hline \multirow{2}{*}{$\mathrm{PPI}+\mathrm{C}+\mathrm{A}$} & Statins & $605(32)$ & $541(83)$ & $79-86$ & \multirow[b]{2}{*}{0.063} & $531(83)$ & $79-86$ & \multirow{2}{*}{0.048} & $567(15)$ & $12-18$ & \multirow[b]{2}{*}{0.792} \\
\hline & No S & 2259 (39) & $1779(86)$ & $84-87$ & & $1753(86)$ & $85-88$ & & 1985 (15) & $14-17$ & \\
\hline \multirow{2}{*}{$\mathrm{PPI}+\mathrm{C}+\mathrm{A}+\mathrm{M} / \mathrm{T}$ conc } & Statins & $541(29)$ & $532(91.5)$ & 89-94 & \multirow{2}{*}{0.056} & $526(92)$ & 89-94 & \multirow{2}{*}{0.099} & $537(19)$ & $16-22$ & \multirow{2}{*}{0.004} \\
\hline & No $S$ & $1253(21)$ & $1207(88.5)$ & $87-90$ & & $1189(89)$ & $87-91$ & & $1229(25)$ & $23-28$ & \\
\hline \multirow{2}{*}{$P P I+C+A+B$} & Statins & $186(10)$ & $182(92)$ & $87-96$ & \multirow[b]{2}{*}{0.131} & $177(92)$ & $87-96$ & \multirow[b]{2}{*}{0.180} & $181(30)$ & $24-38$ & \multirow{2}{*}{0.094} \\
\hline & No $S$ & $840(14)$ & $610(88)$ & $86-91$ & & $603(89)$ & $86-91$ & & $824(24)$ & $22-28$ & \\
\hline \multirow{2}{*}{ PPI + single capsule * } & Statins & $291(16)$ & $279(95)$ & $92-98$ & \multirow{2}{*}{0.540} & $275(96)$ & $93-98$ & \multirow{2}{*}{0.700} & $283(26)$ & $21-32$ & \multirow{2}{*}{0.067} \\
\hline & No $S$ & $636(11)$ & $601(94)$ & $92-96$ & & $590(95)$ & $93-97$ & & $612(32)$ & $29-36$ & \\
\hline \multirow{2}{*}{$\mathrm{PPI}+\mathrm{C}+\mathrm{M} / \mathrm{T}$} & Statins & $99(5)$ & $93(80)$ & $70-87$ & \multirow[b]{2}{*}{0.660} & $93(80)$ & $70-87$ & \multirow[b]{2}{*}{0.657} & $94(19)$ & $12-29$ & \multirow[b]{2}{*}{0.852} \\
\hline & No S & $441(8)$ & $384(81.5)$ & $77-85$ & & $382(82)$ & $77-85$ & & $395(20)$ & $16-24$ & \\
\hline $\mathrm{PPI}+\mathrm{C}+\mathrm{A}+\mathrm{M} / \mathrm{T}$ seq & Statins & $45(2)$ & $41(90)$ & 77-97 & 0.566 & $40(90)$ & $76-97$ & 0.566 & $42(7)$ & $2-20$ & 0.066 \\
\hline \multirow[b]{2}{*}{$\mathrm{PPI}+\mathrm{L}+\mathrm{A}$} & Statins & $24(1)$ & $23(78)$ & $56-93$ & & $23(78)$ & $56-93$ & & $24(29)$ & $13-51$ & \\
\hline & No S & $79(1)$ & $75(88)$ & $78-94$ & 0.243 & $74(88)$ & $78-94$ & 0.254 & $75(21)$ & $13-32$ & 0.429 \\
\hline & Statins & $13(1)$ & $13(92)$ & 64-100 & & $13(92)$ & 64-100 & & $13(31)$ & $9-61$ & \\
\hline$P P I+M / T+A$ & No S & $55(1)$ & 47 (91.5) & $80-98$ & 1 & 47 (91.5) & $80-98$ & 1 & $55(25.5)$ & $15-39$ & 0.733 \\
\hline
\end{tabular}

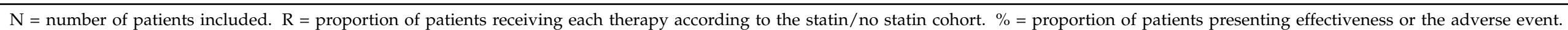

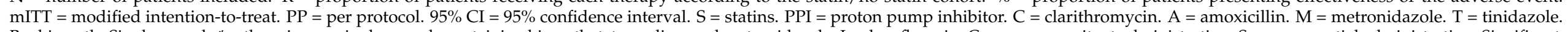

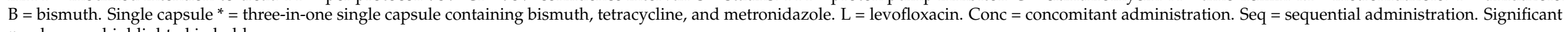
$p$-values are highlighted in bold. 
Table 6. Multivariate analysis of mITT effectiveness in first-line empirically prescribed therapies.

\begin{tabular}{|c|c|c|c|c|c|c|c|c|c|c|c|c|c|c|c|c|c|c|c|}
\hline & & \multicolumn{3}{|c|}{ Overall } & \multicolumn{3}{|c|}{$\mathrm{PPI}+\mathrm{C}+\mathrm{A}$} & \multicolumn{3}{|c|}{$\mathrm{PPI}+\mathrm{C}+\mathrm{M} / \mathrm{T}$} & \multicolumn{3}{|c|}{$\mathrm{PPI}+\mathrm{C}+\mathrm{A}+\mathrm{M} / \mathrm{T}$ Conc } & \multicolumn{3}{|c|}{$\mathrm{PPI}+\mathrm{C}+\mathrm{A}+\mathrm{B}$} & \multicolumn{3}{|c|}{ PPI + Single Capsule * } \\
\hline & & OR & $95 \% \mathrm{CI}$ & $p$-Value & OR & $95 \% \mathrm{CI}$ & $p$-Value & OR & $95 \% \mathrm{CI}$ & $p$-Value & OR & $95 \% \mathrm{CI}$ & $p$-Value & OR & $95 \%$ CI & $p$-Value & OR & $95 \% \mathrm{CI}$ & $p$-Value \\
\hline Gender & $\begin{array}{c}\text { Female } \\
\text { Male }\end{array}$ & $\begin{array}{c}1 \\
1.367\end{array}$ & $1.2-1.6$ & $<0.001$ & 1.547 & $1.2-1.9$ & 0.001 & & NS & & & NS & & & NS & & & NS & \\
\hline PPI dose & $\begin{array}{l}\text { Low } \\
\text { Standard } \\
\text { High }\end{array}$ & $\begin{array}{c}1 \\
1.619 \\
1.982\end{array}$ & $\begin{array}{c}1.3-2 \\
1.6-2.4\end{array}$ & $\begin{array}{l}<0.001 \\
<0.001\end{array}$ & $\begin{array}{l}1.843 \\
2.644\end{array}$ & $\begin{array}{l}1.4-2.4 \\
1.9-3.6\end{array}$ & $\begin{array}{l}<0.001 \\
<0.001\end{array}$ & $\begin{array}{l}3.698 \\
2.518\end{array}$ & $\begin{array}{c}1.2-12 \\
1.4-4.7\end{array}$ & $\begin{array}{l}0.026 \\
0.003\end{array}$ & $\begin{array}{c}1 \\
1.381 \\
1.827\end{array}$ & $\begin{array}{c}0.92-2.1 \\
1.3-2.6\end{array}$ & $\begin{array}{l}0.122 \\
0.001\end{array}$ & $\begin{array}{c}1 \\
7.451 \\
2.451\end{array}$ & $\begin{array}{c}3.0-18 \\
1.5-4.0\end{array}$ & $\begin{array}{l}<0.001 \\
<0.001\end{array}$ & & NS & \\
\hline Statin use & $\begin{array}{l}\text { No } \\
\text { Yes }\end{array}$ & & NS & & 0.763 & $0.59-0.99$ & 0.046 & & NS & & & NS & & & NS & & & NS & \\
\hline
\end{tabular}

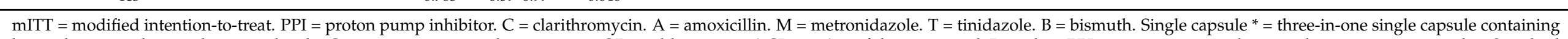

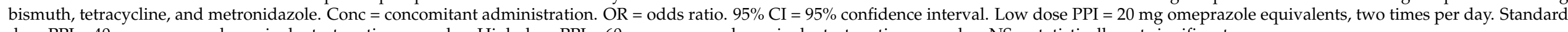
dose PPI $=40 \mathrm{mg}$ omeprazole equivalents, two times per day. High dose PPI $=60 \mathrm{mg}$ omeprazole equivalents, two times per day. NS $=$ statistically not significant 
The standard triple regimen containing a PPI, clarithromycin, and amoxicillin, and the non-bismuth quadruple concomitant therapy (PPI-clarithromycin-amoxicillin-nitroimidazole), were the therapies most frequently prescribed in both groups.

The sub-analysis by therapy revealed that the highest eradication rates were obtained with the single capsule bismuth quadruple therapy (containing tetracycline, metronidazole, and bismuth salts in a single capsule administered together with a PPI), the bismuthamoxicillin-clarithromycin quadruple therapy (adding these antibiotics to a PPI), and the non-bismuth concomitant regimen (PPI-amoxicillin-clarithromycin-nitroimidazole); all of them exhibited close to $90 \%$ mITT effectiveness (Figure 2). Similar results were obtained for these therapies in statin users and non-users, with non-significant differences between both cohorts. However, the statin users who received the concomitant quadruple regimen showed a tendency towards higher effectiveness than non-users that was close to statistical significance $(\mathrm{OR}=1.4 ; 95 \% \mathrm{CI}: 1-2 ; p=0.059)$. Only the standard triple therapy showed a significant difference in effectiveness according to the statin status, with lower effectiveness in statin users (OR = 0.763; 95\% CI: 0.59-0.99; $p<0.05)$ (Tables 5 and 6).

Regarding safety, the overall incidence of AEs was similar between both groups (20\% in statin users vs. $21 \%$ in non-users), and most of them were mild (Figure 3 ). However, the rate of AEs was significantly different between users and non-users in the concomitant quadruple therapy group specifically (19\% vs. 25\%, $p<0.05$ ) (Table 5, Figure 3, online Supplementary Table S3). The incidence rate of serious AEs (SAEs) was $<1 \%$ in both groups. In the statin users group, one patient experienced diarrhea with disability after using the concomitant quadruple therapy. In the non-users cohort, three patients presented an SAE, all after the single capsule bismuth therapy. These events were: an episode of acute pancreatitis, a process of abdominal pain and vomiting, both requiring hospitalization, and a third event that was not explained in the database.

The specific analysis evaluating effectiveness or safety of eradication therapies according to the different statins used (simvastatin, atorvastatin, rosuvastatin, and other statins) showed no differences, neither in the overall group nor when considering each therapy separately (online Supplementary Table S4).

\subsubsection{Rescue Therapies}

A total of 2228 patients received an empirical rescue therapy after failing at least one treatment attempt to eradicate $H$. pylori infection, $1612(72 \%)$ received a second-line therapy, $459(21 \%)$ a third-line, $111(5 \%)$ a fourth-line, $34(1.5 \%)$ a fifth-line, and $12(0.5 \%)$ a sixth-line.

Likewise, two cohorts could be distinguished: statin users $(n=589)$ and non-users $(n=1639)$. Overall mITT effectiveness was found to be different in these two cohorts: $87 \%$ in the statin-users and $78 \%$ in non-users $(p<0.001)$ (Table 7$)$. The multivariate analysis showed statin-use as a variable being significantly associated with higher mITT effectiveness $(\mathrm{OR}=1.9 ; 95 \% \mathrm{CI}: 1.4-2.6 ; p<0.001)$. See Table 8 for detailed results of the multivariate analysis.

The triple therapy containing a PPI, amoxicillin, and levofloxacin, followed by the single capsule bismuth quadruple therapy, and the bismuth-amoxicillin-levofloxacin quadruple therapy (adding these antibiotics to a PPI) were the three most frequently prescribed therapies in both cohorts (Table 7).

The sub-analysis performed specifically on each therapy revealed that the best mITT effectiveness result was achieved both with the single capsule bismuth and the bismuthamoxicillin-levofloxacin quadruple therapies (close to $90 \%$; Figure 2). The presence of statins was significantly associated with higher effectiveness only in the single capsule bismuth treatment $(\mathrm{OR}=2.8 ; 95 \% \mathrm{CI}: 1.3-5.7 ; p<0.05)$. No association between effectiveness and statin use was found within the remaining therapies (Table 8). 
A

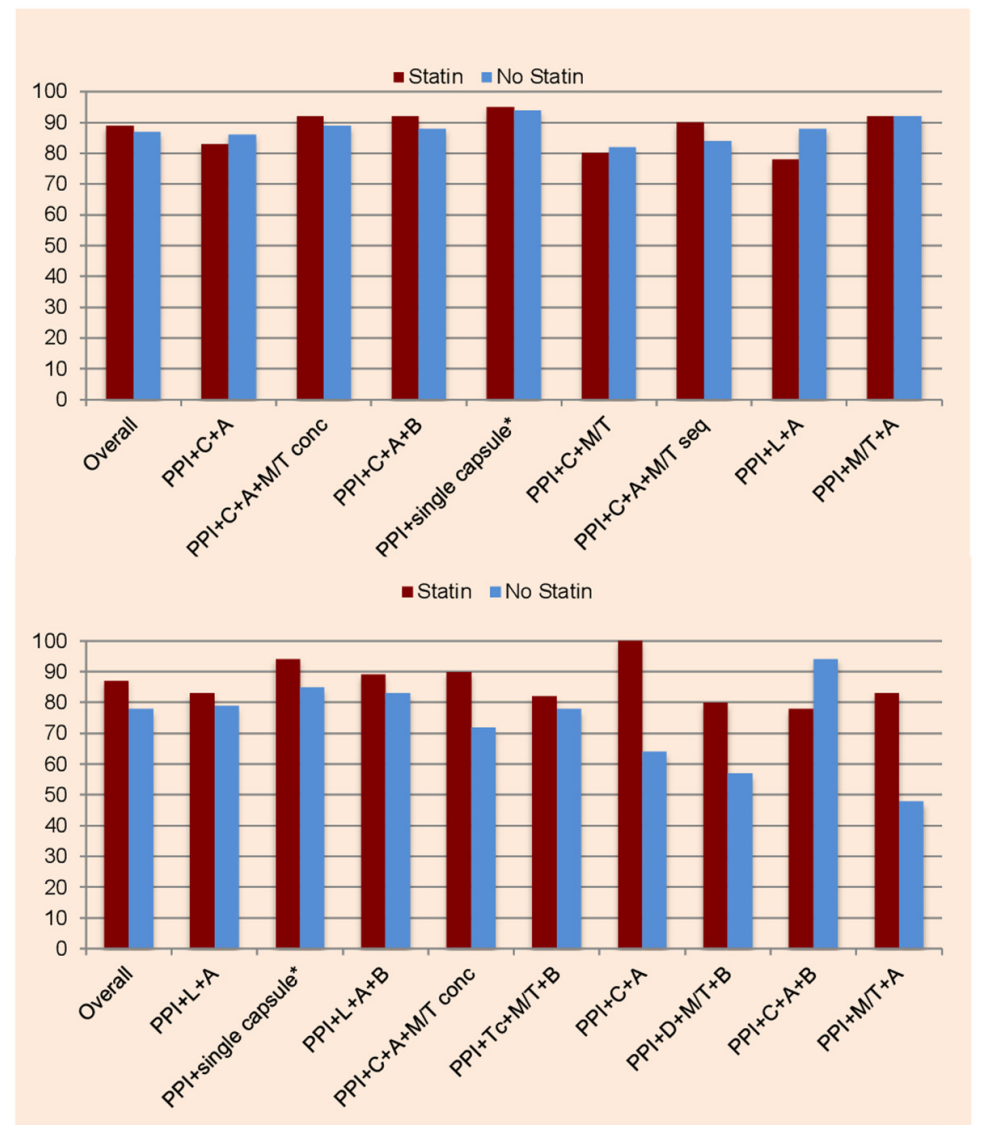

C

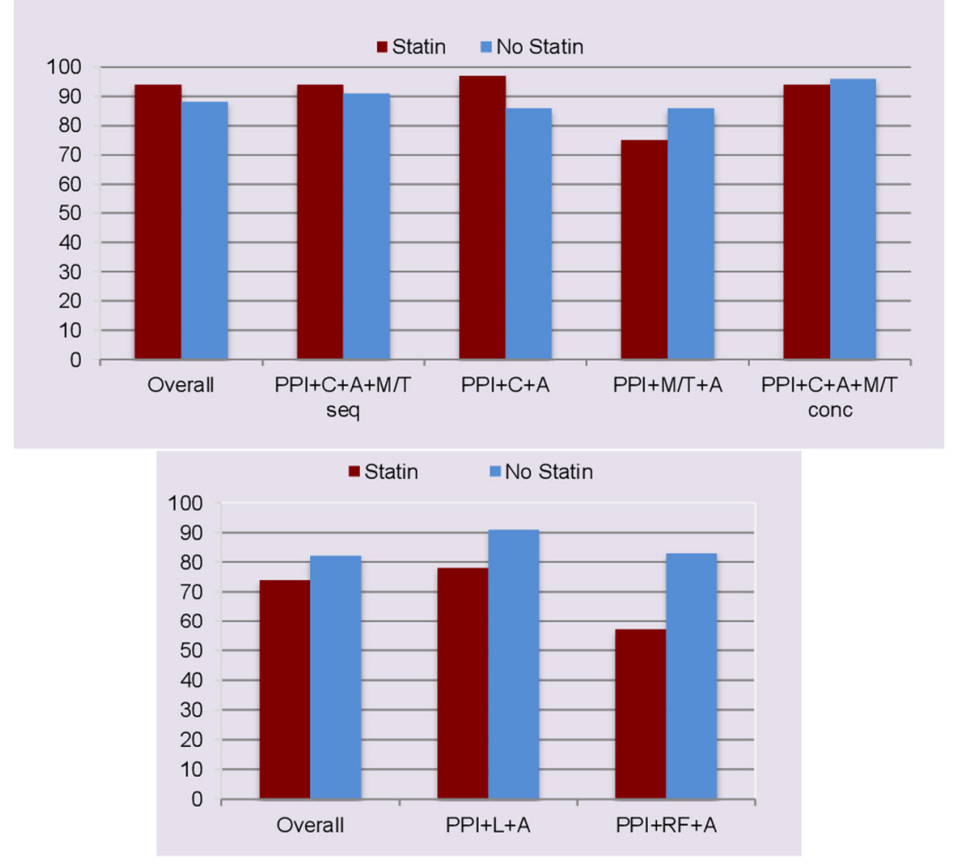

Figure 2. mITT effectiveness according to statin-status and receiving each specific therapy. (A) Empirical approach: first-line. (B) Empirical approach: rescue lines. (C) Culture-guided approach: first-line. (D) Culture-guided approach: rescue lines. PPI = proton pump inhibitor. $\mathrm{C}=$ clarithromycin. $\mathrm{A}=$ amoxicillin. $\mathrm{M}=$ metronidazole. $\mathrm{T}=$ tinidazole $\mathrm{B}=$ bismuth. Single-capsule ${ }^{*}=$ three-in-one single capsule containing bismuth, tetracycline and metronidazole. $\mathrm{L}=$ levofloxacin. $\mathrm{Tc}=$ tetracycline. $\mathrm{D}=$ doxycycline. $\mathrm{RF}=$ rifabutine. Conc $=$ concomitant administration. $\mathrm{Seq}=$ sequential administration . 


\section{Empirical prescriptions}
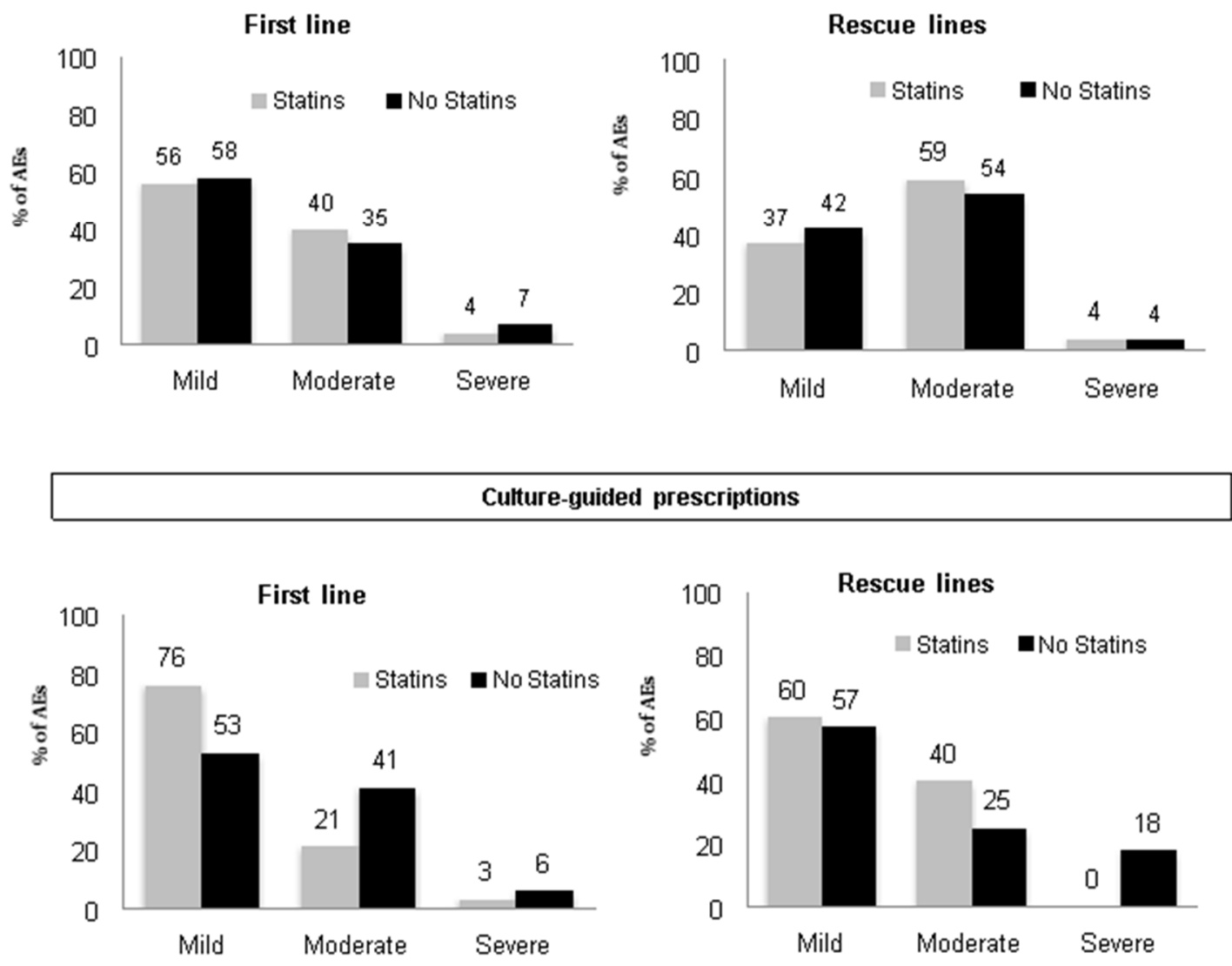

Figure 3. Distribution of adverse event severity presented according to statin use and treatment line.

The overall incidence of AEs was higher in statin users than in non-users (35\% vs. $28 \%, p<0.05$ ), and most of them were moderate (Figure 3). Only the bismuth-amoxicillinclarithromycin quadruple therapy showed a significantly higher rate of AE in the statin cohort than in non-users; however, since the sample size of both cohorts was very small, this finding remains non-conclusive. See Table 7, Figure 3 and online Supplementary Table S3 for a more specific analysis.

SAEs were present only in four patients $(<1 \%)$, all of them in the non-statin users cohort: one patient presented severe abdominal pain requiring hospitalization (after using the standard triple therapy), one patient presented an episode of severe diarrhea (after the triple amoxicillin-levofloxacin therapy), one patient a Clostridioides difficile infection (after the single capsule bismuth quadruple), and the last patient presented nausea, diarrhea, weight loss, and laboratory abnormalities (after taking a marginal regimen).

The specific analysis according to the different statins used (simvastatin, atorvastatin, rosuvastatin, and other statins) showed, again, no differences both in the overall group and considering each therapy separately. A detailed analysis is shown in online Supplementary Table S4. 
Table 7. Effectiveness and safety in rescue lines empirically prescribed therapies: 2nd-6th lines.

\begin{tabular}{|c|c|c|c|c|c|c|c|c|c|c|c|}
\hline & & \multirow[t]{2}{*}{$\mathbf{N}(\mathbf{R})$} & \multicolumn{3}{|c|}{ mITT Effectiveness } & \multicolumn{3}{|c|}{ PP Effectiveness } & \multicolumn{3}{|c|}{ Adverse Events } \\
\hline & & & N (\%) & $95 \%$ CI & $p$-Value & N (\%) & $95 \% \mathrm{CI}$ & $p$-Value & N (\%) & $95 \% \mathrm{CI}$ & $p$-Value \\
\hline \multirow{2}{*}{ Overall } & Statins & 589 & $565(87)$ & $84-90$ & \multirow{2}{*}{$<0.001$} & $549(88)$ & $85-91$ & \multirow{2}{*}{$<0.001$} & $568(35)$ & $31-39$ & \multirow{2}{*}{0.003} \\
\hline & No S & 1639 & $1480(78)$ & $76-80$ & & $1449(78)$ & $76-80$ & & $1542(28)$ & $26-31$ & \\
\hline \multirow{2}{*}{$\mathrm{PPI}+\mathrm{L}+\mathrm{A}$} & Statins & $177(30)$ & $172(83)$ & $76-88$ & \multirow{2}{*}{0.263} & $169(83)$ & $76-88$ & \multirow{2}{*}{0.275} & $175(27)$ & $21-35$ & \multirow{2}{*}{0.584} \\
\hline & NoS & $545(33)$ & $504(79)$ & $75-82$ & & $494(79)$ & $75-83$ & & $521(25)$ & $22-29$ & \\
\hline \multirow{2}{*}{ PPI + single capsule * } & Statins & $170(29)$ & $164(94)$ & $89-97$ & \multirow{2}{*}{0.006} & $159(95)$ & $90-98$ & \multirow{2}{*}{0.002} & $165(42)$ & $35-50$ & \multirow{2}{*}{0.473} \\
\hline & No S & $328(20)$ & $298(85)$ & $81-89$ & & $291(86)$ & $81-89$ & & $310(39)$ & $34-45$ & \\
\hline $\mathrm{PPI}+\mathrm{L}+\mathrm{A}+\mathrm{B}$ & No S & $178(11)$ & $166(83)$ & $77-89$ & 0.280 & $164(83.5)$ & $77-89$ & 0.133 & $168(34.5)$ & $27-42$ & 0.556 \\
\hline \multirow{2}{*}{$\mathrm{PPI}+\mathrm{C}+\mathrm{A}+\mathrm{M} / \mathrm{T}$ conc } & Statins & $31(5)$ & $31(90)$ & $74-98$ & \multirow[b]{2}{*}{0.043} & $30(90)$ & $74-98$ & \multirow{2}{*}{0.107} & $31(29)$ & $14-48$ & \multirow{2}{*}{0.253} \\
\hline & NoS & $76(5)$ & $71(72)$ & $60-82$ & & $68(73.5)$ & $61-84$ & & $74(19)$ & $11-30$ & \\
\hline \multirow{2}{*}{$\mathrm{PPI}+\mathrm{M} / \mathrm{T}+\mathrm{T} \mathrm{c}+\mathrm{B}$} & Statins & $17(3)$ & $17(82)$ & $57-96$ & \multirow{2}{*}{1} & $17(82)$ & $57-96$ & \multirow[b]{2}{*}{1} & $17(35)$ & $14-62$ & \multirow[b]{2}{*}{0.933} \\
\hline & No S & $88(5)$ & $83(78)$ & $68-87$ & & $82(78)$ & $68-86$ & & $88(36)$ & $26-47$ & \\
\hline \multirow[b]{2}{*}{$\mathrm{PPI}+\mathrm{C}+\mathrm{A}$} & Statins & $10(2)$ & $10(100)$ & NA & \multirow[b]{2}{*}{0.024} & $10(100)$ & NA & \multirow[b]{2}{*}{0.024} & $10(20)$ & $3-56$ & \multirow[b]{2}{*}{1} \\
\hline & No S & $53(3)$ & $44(64)$ & $48-78$ & & $44(64)$ & $48-78$ & & $52(23)$ & $13-37$ & \\
\hline $\mathrm{PPI}+\mathrm{M} / \mathrm{I}+\mathrm{D}+\mathrm{B}$ & No S & $44(3)$ & $42(57)$ & $41-72$ & 0.134 & $41(56)$ & $40-72$ & 0.205 & $43(23)$ & $12-39$ & 0.728 \\
\hline & Statins & $10(2)$ & $9(78)$ & $40-97$ & & $9(78)$ & $40-97$ & & $9(56)$ & $21-86$ & \\
\hline $\mathrm{PPI}+\mathrm{C}+\mathrm{A}+\mathrm{B}$ & NoS & $40(2)$ & $34(94)$ & $80-99$ & 0.188 & $33(97)$ & $84-100$ & 0.111 & $36(19)$ & $8-36$ & 0.043 \\
\hline & Statins & $6(1)$ & $6(83)$ & $36-100$ & & $6(83)$ & $36-100$ & & $6(0)$ & 0 & \\
\hline $\mathrm{PPI}+\mathrm{M} / \mathrm{T}+\mathrm{A}$ & NoS & $35(2)$ & $29(48)$ & $30-68$ & 0.187 & $29(48)$ & $30-68$ & 0.187 & $31(6.5)$ & $1-21$ & 1 \\
\hline
\end{tabular}

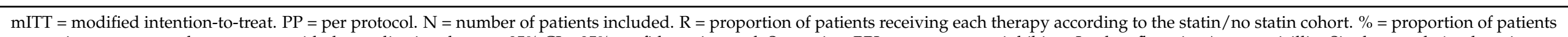

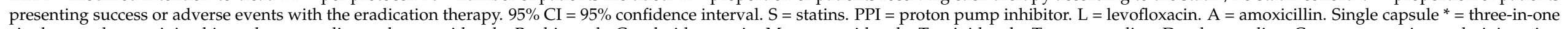

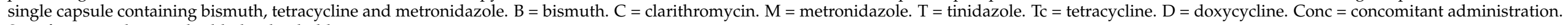
Significant $p$-values are highlighted in bold. 
Table 8. Multivariate analysis of mITT effectiveness in rescue attempts according to empirically prescribed therapies: 2nd-6th lines.

\begin{tabular}{|c|c|c|c|c|c|c|c|c|c|c|c|c|c|c|c|c|c|c|c|}
\hline & & \multicolumn{3}{|c|}{ Overall } & \multicolumn{3}{|c|}{ PPI + L+A } & \multicolumn{3}{|c|}{ PPI + Single Capsule * } & \multicolumn{3}{|c|}{$\mathrm{PPI}+\mathrm{L}+\mathrm{A}+\mathrm{B}$} & \multicolumn{3}{|c|}{$\mathrm{PPI}+\mathrm{C}+\mathrm{A}+\mathrm{M} / \mathrm{T}$ Conc } & \multicolumn{3}{|c|}{$\mathrm{PPI}+\mathrm{M} / \mathrm{T}+\mathrm{Tc}+\mathrm{B}$} \\
\hline & & OR & $95 \%$ CI & $p$-Value & OR & $95 \%$ CI & $p$-Value & OR & $95 \%$ CI & $p$-Value & OR & $95 \%$ CI & $p$-Value & OR & $95 \%$ CI & $p$-Value & OR & $95 \%$ CI & $p$-Value \\
\hline Age (years) & $\begin{array}{c}18-30 \\
31-50 \\
51-70 \\
>70\end{array}$ & $\begin{array}{c}1 \\
0.413 \\
0.357 \\
0.442\end{array}$ & $\begin{array}{l}0.20-0.84 \\
0.18-0.72 \\
0.20-0.96\end{array}$ & $\begin{array}{l}0.015 \\
0.004 \\
0.039\end{array}$ & & NS & & & NS & & & NS & & & NS & & & NS & \\
\hline Diagnosis & $\begin{array}{c}\text { No ulcer } \\
\text { Ulcer disease }\end{array}$ & $\begin{array}{c}1 \\
1.520 \\
\end{array}$ & $1.1-2.1$ & 0.008 & & NS & & & NS & & & NS & & & NS & & & NS & \\
\hline Length (days) & $\begin{array}{c}7 \\
10 \\
14 \\
\end{array}$ & $\begin{array}{c}1 \\
2.425 \\
2.914 \\
\end{array}$ & $\begin{array}{l}1.4-4.2 \\
1.6-5.2 \\
\end{array}$ & $\begin{array}{c}0.001 \\
<0.001 \\
\end{array}$ & $\begin{array}{c}1 \\
2.818 \\
\end{array}$ & $1.6-5.0$ & $<0.001$ & & NS & & & NS & & & NS & & $\begin{array}{c}1 \\
1.246 \\
5.640 \\
\end{array}$ & $\begin{array}{c}0.26-6.0 \\
1.0-31 \\
\end{array}$ & $\begin{array}{l}0.784 \\
0.046 \\
\end{array}$ \\
\hline PPI dose & $\begin{array}{c}\text { Low } \\
\text { Standard } \\
\text { High }\end{array}$ & $\begin{array}{c}1 \\
1.204 \\
1.995 \\
\end{array}$ & $\begin{array}{c}0.89-1.6 \\
1.5-2.7 \\
\end{array}$ & $\begin{array}{c}0.230 \\
<0.001 \\
\end{array}$ & $\begin{array}{c}1 \\
1.283 \\
1.921 \\
\end{array}$ & $\begin{array}{c}0.80-2.1 \\
1.1-3.4\end{array}$ & $\begin{array}{l}0.296 \\
0.024 \\
\end{array}$ & & NS & & $\begin{array}{c}1 \\
2.778 \\
3.822 \\
\end{array}$ & $\begin{array}{c}0.43-18 \\
1.2-12 \\
\end{array}$ & $\begin{array}{l}0.284 \\
0.025 \\
\end{array}$ & & NS & & & NS & \\
\hline Compliance & $\begin{array}{l}\text { No } \\
\text { Yes }\end{array}$ & $\begin{array}{c}1 \\
3.937 \\
\end{array}$ & $2.0-7.6$ & $<0.001$ & & NS & & $\begin{array}{c}1 \\
4.442 \\
\end{array}$ & $1.3-15.8$ & 0.021 & $\begin{array}{c}1 \\
6.880 \\
\end{array}$ & $1.3-36$ & 0.022 & & NS & & & NS & \\
\hline Statin use & $\begin{array}{l}\text { No } \\
\text { Yes }\end{array}$ & $\begin{array}{c}1 \\
1.896\end{array}$ & $1.4-2.6$ & $<0.001$ & & NS & & $\begin{array}{c}1 \\
2.752\end{array}$ & $1.3-5.7$ & 0.006 & & NS & & $\begin{array}{c}1 \\
3.660\end{array}$ & $0.99-13$ & 0.050 & & NS & \\
\hline
\end{tabular}

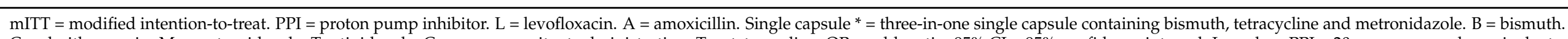

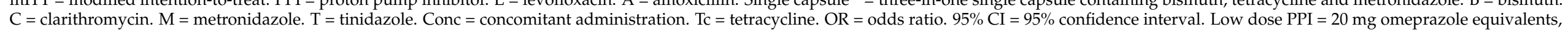
two times per day. Standard dose PPI $=40 \mathrm{mg}$ omeprazole equivalents, two times per day. High dose PPI $=60 \mathrm{mg}$ omeprazole equivalents, two times per day. NS $=$ statistically not significant. 


\subsection{Culture-Guided Prescriptions}

In total, 705 patients received an eradication therapy guided by the antibiotic resistance profile. Unlike what happened with the empirical group, the effectiveness was similar between statin users and non-users $(90 \%$ vs. $87 \% ; p=0.27$ in the univariate analysis; $\mathrm{OR}=1.3 ; 95 \%$ CI: $0.71-2.4 ; p=0.378$ in the multivariate analysis). The only variables included in the multivariate analysis that showed significant results in terms of mITT effectiveness were the administration of a first-line instead of a rescue treatment approach, and good compliance (Table 4). No differences were observed in the rate of AEs between both groups ( $22 \%$ vs. $20 \%$ for statin users and non-users, respectively, $p=0.589)$ (Table 3 ). Detailed analyses by treatment scheme in first-line and rescue eradication therapy are described below.

\subsubsection{First-Line Therapy}

A total of 531 patients received a first-line therapy: 130 were statin users and 401 were non-users. No differences concerning the effectiveness were found between both cohorts (93.5\% for statins vs. $88 \%$ for non-users; $p=0.095)$. The multivariate analysis showed no significant differences in mITT effectiveness results according to the statin-status (OR $=1.9$; 95\% CI: 0.85-4.1; $p=0.120$ ) (Tables 9 and 10).

The non-bismuth quadruple sequential therapy (PPI-clarithromycin-amoxicillinnitroimidazole administered sequentially), and the standard triple therapy were the most frequently prescribed therapies in both groups. Both therapies showed a success higher than $90 \%$. No differences in effectiveness or safety were found between the presence or absence of statins for each specific therapy (Tables 9 and 10, and Figure 2).

According to the overall safety, both groups experienced similar rates of AEs ( $21 \% \mathrm{vs.}$ $22 \%$ ), which were mostly of mild intensity (Figure 3). No SAEs were reported.

The specific analysis according to the different statins used (simvastatin, atorvastatin, rosuvastatin, and other statins) showed no association between any of them and the effectiveness or safety of the eradication therapies, either globally or in the sub-analyses of the main therapies used (online Supplementary Table S5).

\subsubsection{Rescue Therapies}

A total of 173 patients received a rescue therapy guided by culture, $74(43 \%)$ received a second attempt, $65(38 \%)$ a third attempt, $19(11 \%)$ a fourth attempt, $11(6 \%)$ a fifth attempt, and $4(2 \%)$ a sixth-line eradication attempt. Of these, 33 were statin users and 140 were non-users.

mITT effectiveness was similar in statin users and non-users in the univariate analysis $(74 \%$ vs. $82 \% ; p=0.379)$. No association was found between effectiveness and any of the other variables included in the multivariate analysis. Safety findings were also similar (Figure 3).

The majority of patients in both groups received a triple levofloxacin-amoxicillin therapy or a triple therapy adding rifabutine and amoxicillin to a PPI. No differences in mITT effectiveness were found to be significant between statin users and non-users (Tables 9 and 10, and Figure 2), or between the use of simvastatin vs. atorvastatin (online Supplementary Table S5). 
Table 9. Effectiveness and safety in culture-guided eradication therapies.

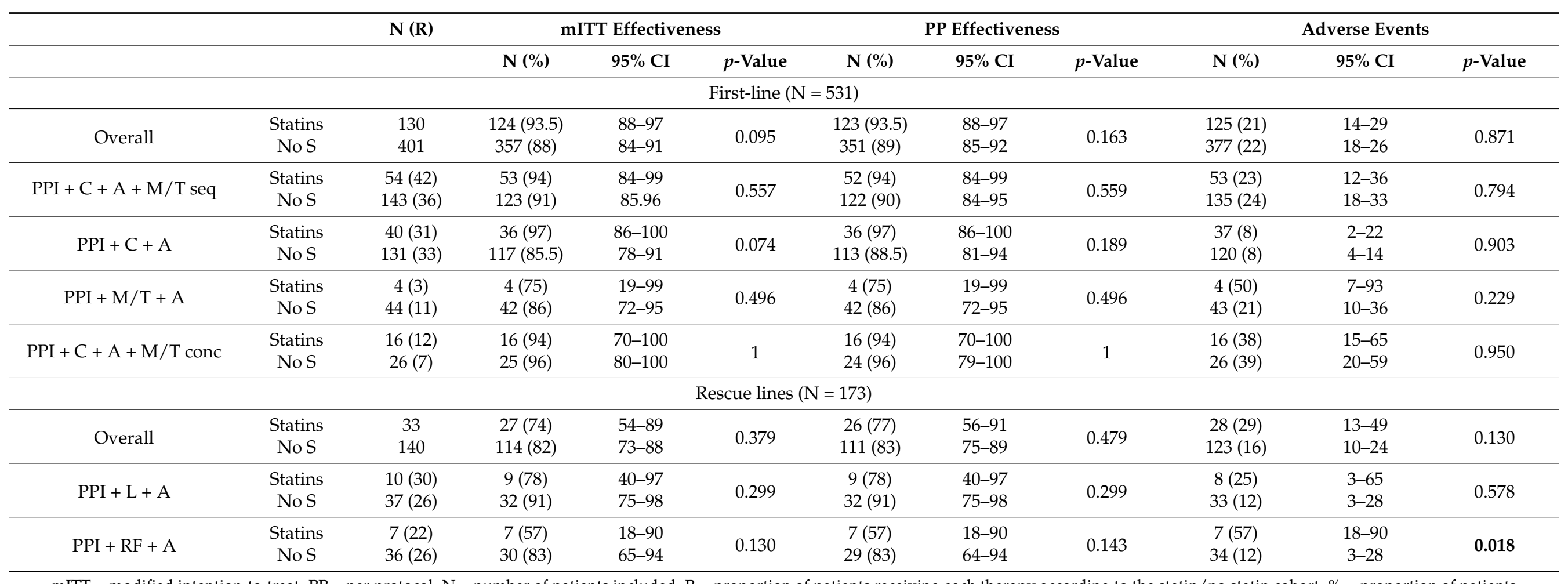

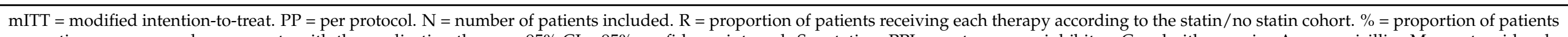

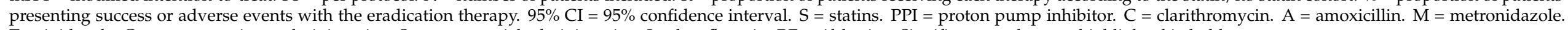
$\mathrm{T}=$ tinidazole. Conc $=$ concomitant administration. $\mathrm{Seq}=$ sequential administration. $\mathrm{L}=$ levofloxacin. $\mathrm{RF}=$ rifabutine. Significant $p$-values are highlighted in bold. 
Table 10. Multivariate analysis of mITT effectiveness according to culture-guided prescribed therapies.

\begin{tabular}{ccccccc}
\hline & \multicolumn{3}{c}{ First-Line } & \multicolumn{3}{c}{ Rescue Therapies } \\
\hline & OR & $\mathbf{9 5 \%}$ CI & $p$-Value & OR & $\mathbf{9 5 \%}$ CI & $p$-Value \\
\hline $\begin{array}{c}\text { Compliance } \\
\text { No } \\
\text { Yes }\end{array}$ & 1 & & & & \\
\hline
\end{tabular}

mITT = modified intention-to-treat. Rescue therapies = embracing therapies prescribed from 2nd-6th eradication attempt. OR = odds ratio. $95 \% \mathrm{CI}=95 \%$ confidence interval. NS = non statistically significant.

\section{Discussion}

The present study is a sub-analysis of the Hp-EuReg focused on evaluating whether the use of statins could modify the effectiveness or the safety of the therapies prescribed against $H$. pylori. It is important to clarify that statins had already been initiated in our study when the eradication regimen was prescribed.

We separately evaluated empirical and culture-guided prescriptions of eradication therapies, differentiating within each group between the first treatment approach and the rescue attempts, as both conditions are considered to modify per se the effectiveness of H. pylori treatment.

Patients receiving an empirically prescribed first-line therapy showed optimal effectiveness rates independently of their statin-status (close to $90 \%$ in both cases). Other parameters such as the use of longer eradication therapies, use of standard or high PPI doses, or good compliance were associated with higher effectiveness. These associations had already been observed in previous reports, which reinforces our findings [30-32].

The specific analysis of first-line therapies showed that the single capsule bismuth quadruple, the bismuth-amoxicillin-clarithromycin quadruple, and the non-bismuth concomitant regimen obtained the highest effectiveness, in agreement with previous analyses $[7,33,34]$. Neither of these therapies showed an association between treatment effectiveness and the use of statins. Only in the standard triple therapy was the use of these drugs associated with lower effectiveness. This finding is opposed to what was described in the randomized studies of Nseir and Hassan, in which the addition of simvastatin $20 \mathrm{mg}$ twice daily to the standard triple therapy containing clarithromycin and amoxicillin showed a statistically significant increase in effectiveness of 15-20\% [26,35]. Despite their findings, several voices questioned whether this hypothetical increase would be clinically significant considering the unacceptable effectiveness rate reported for the standard triple therapy over the years in most geographical areas $[1,6,36]$.

In the group of patients receiving an empirical rescue treatment, the use of statins was associated with higher effectiveness in the overall cohort. Other factors that showed an association with higher effectiveness were peptic ulcer diagnosis, younger age of the patients, use of longer therapies, use of high doses of PPIs, and good compliance. However, the specific sub-analysis of the main eradication therapies only revealed an association between the use of statins and the effectiveness of the single capsule bismuth quadruple therapy. No previous evidence concerning this association had been published before. However, evidence evaluating another quadruple therapy, the bismuth-amoxicillinclarithromycin quadruple therapy, had previously provided conflicting results: Parsi and collaborators evaluated the effectiveness of adding simvastatin (10 or $20 \mathrm{mg}$ once daily) to a 14-day treatment with this quadruple therapy and found no significant differences in treatment effectiveness between the statin and placebo groups, with the effectiveness being around $90 \%$ in both cases [37]. On the other hand, Sarkeshikian and collaborators found significantly better results with the addition of atorvastatin $40 \mathrm{mg}$ once daily to 14-day bismuth-amoxicillin-clarithromycin quadruple therapy (increasing effectiveness from 65\% to $78 \%$ ) [38]. Although this increase in effectiveness might seem relevant, the effectiveness values obtained in both cases can be considered suboptimal; accordingly, possible factors explaining these insufficient results should be carefully addressed. 
Therefore, the results obtained in our study concerning statins' role in eradication therapy were not homogeneous in first or rescue lines, and neither were they when evaluating the different eradication treatment schemes. Up to now, the exact role of this hypothetical synergism between drugs based on a pure anti-infection role has not been well elucidated or universally proven. Some authors have shown that cholesterol cellular depletion caused by statins promotes autophagy in macrophages containing $H$. pylori, producing a drop in the burden of these bacteria. However, other groups failed to show this descent in bacterial viability, so more studies are needed to uncover the real effect of this association in eradication terms of the infection [24,27]. The role of the statins in decreasing the inflammatory response caused by Cag A protein from $\mathrm{H}$. pylori in gastric mucosa, by lowering cellular cholesterol levels and decreasing NF- $K \beta$ and IL- 8 has also been reported, with encouraging results in gastric cancer. Again, large scale studies need to be performed to evaluate this fact before global conclusions can be drawn [22,28].

Concerning safety parameters, the addition of statins to the eradication therapy was not associated with significant changes in AE rates (which appeared in around 20-25\% of the patients). Only in the rescue-attempt group was a higher rate of AEs observed in statin-users compared to non-users ( $35 \%$, vs. $28 \%)$, although this difference was not confirmed when addressing each therapy separately. In general terms, most of the AEs were mild, and the rate of SAEs was marginal in both cohorts.

Finally, no specific differences in effectiveness or safety were found according to the different statins used: simvastatin vs. atorvastatin vs. rosuvastatin vs. other minority statins. Other studies divided statins in two groups categorized as high or low potency, considering the type of drug and its dosage, as well as the magnitude of the decrease induced in basal LDL-cholesterol ( $\geq 45 \%$ or $<45 \%$, respectively) [39].

Our study has several limitations. The main one is its design as an observational non-interventional study. As such, statin use was not randomized, with consequent heterogeneous cohorts for certain demographical variables and the resulting potential risk of bias. In spite of this limitation, we believe this design provides interesting results coming from a real-practice European setting, which are, therefore, more easily applicable to daily clinical management in this area. Another limitation is the lack of information concerning the exact doses and types of statins used in more than $80 \%$ of patients, which precluded establishing subgroups according to statin potency. This is explained by the initial design of the Hp-EuReg project, which was focused on evaluating H. pylori treatment in routine clinical practice (far from the controlled conditions of experimental settings) rather than studying the relationship between statin types (or their doses) and the eradication regimens, especially considering the lack of previous formal evidence of this association.

In general terms, $\mathrm{Hp}$-EuReg provides a large sample size suitable for evaluating strategies to increase the global effectiveness of the eradication therapies to treat $H$. pylori. Concerning statin use, although combined administration of statins with some therapies was able to modify their effectiveness or safety, this effect was not observed in other lines and /or using other eradication regimens. This lack of a common or consistent behavior raises some concerns about the real utility of adding statins, especially considering that no pathophysiological explanation for the synergistic effect with antibiotics has been unveiled in the framework of $H$. pylori treatment $[27,28]$. It should also be taken into consideration that the addition of another medication to an already complicated-to-follow regimen can affect treatment adherence, which is considered a relevant factor affecting effectiveness. Therefore, the addition of statins to $H$. pylori treatment should not be generally recommended to improve eradication.

\section{Materials and Methods}

The "European Registry on H. pylori Management" (Hp-EuReg) is an international (30 countries), multicenter ( $>300$ investigators), prospective, non-interventional registry that was started in 2013 and was promoted by the European Helicobacter and Microbiota Study Group (www.helicobacter.org, accessed on 6 August 2021). 
The Hp-EuReg protocol was approved by the Ethics Committee of La Princesa University Hospital (Madrid, Spain) [29] and was prospectively registered at ClinicalTrials.gov under the code NCT02328131. The study protocol conforms to the ethical guidelines of the 1975 Declaration of Helsinki as reflected in the approval of the institution's human research committee. Written, informed consent was obtained from each patient included in the study.

Criteria for country selection, national coordinators, and gastroenterologist recruiting investigators are shown in the protocol publication. Monitoring (at least $10 \%$ of the included records in each country and each hospital, respectively), quality of the data, and a list of variables and outcomes are also shown in the same protocol [29].

Data were recorded in an Electronic Case Report Form (e-CRF) and collected and managed using REDCap, a research electronic data capture platform hosted at "Asociación Española de Gastroenterología" (AEG; www.aegastro.es, accessed on 6 August 2021), a non-profit Scientific and Medical Society focused on Gastroenterology research [40,41].

The aim of the current analysis was to evaluate if the concomitant use of statins prescribed for cardiovascular prevention and chronically used could modify the effectiveness rates of $H$. pylori eradication therapies. Secondary aims were directed to assess if this role would be different depending on the type of eradication therapy or statins prescribed and whether these statins would modify AE rates.

For this specific analysis, some quality criteria were applied: only patients with available information on whether they were daily statin users or not were included for evaluation. Exclusion criteria were: patients under the age of 18 years, patients with a period of less than four weeks between the end of the eradication therapy, and patients who were checked for treatment response with the use of serology.

\subsection{Variables}

The e-CRF registered 290 variables containing information about a patient's demographics, comorbidity, indication and method of diagnosis, previous eradication regimens prescribed (if any), current treatment, and data on effectiveness, safety, and compliance. The variable treatment length was assessed using three categories, corresponding to the most frequent treatment durations: 7, 10, and 14 days. Similarly, PPI data were standardized using PPI acid inhibition potency as defined by Kirchheiner [42] and Graham [32], and classified as low, standard and high dose PPIs (online Supplementary File S2). Eradication was confirmed with at least one of the following diagnostic methods: urea breath test, stool antigen test and/or histology.

\subsection{Effectiveness Analysis}

Treatment eradication rate was the main outcome, and was studied in two sets of patients as follows: a modified intention-to-treat (mITT) analysis included all cases that had completed follow-up (that is, with a result of the confirmatory test, either positive or negative) up to January 2020, regardless of compliance. The per-protocol (PP) analysis included all cases that had finished follow-up and had taken at least $90 \%$ of the treatment drugs, as defined in the protocol. Head-to-head comparisons were made between statin and non-statin users for different levels of treatment: first for empirical vs. culture-guided cohorts globally, then for first and rescue eradication attempts (both in empirical and culture-guided cohorts), and finally for each of the most frequently used therapies (only therapies administered to at least 40 patients globally [including both statin users and non-users] were specifically evaluated).

Head-to-head comparisons of treatment effectiveness were made according to the type of statin used: simvastatin, atorvastatin, rosuvastatin, and other statins (including fluvastatin, pitavastatin, pravastatin, and lovastatin). 


\subsection{Safety Analysis}

Head-to-head comparisons of AE incidence were made between statin and non-statin users as follows: first in empirical vs. culture-guided cohorts globally, then in first and rescue eradication attempts (both in empirical and culture-guided cohorts), and finally in each of the most frequently used therapies (only therapies administered to at least 40 patients globally [including both statin users and non-users] were specifically evaluated).

Head-to-head comparisons of safety were also performed overall and considering the aforementioned four statin categories.

\subsection{Statistical Analyses}

Continuous variables were presented as mean and standard deviation. Qualitative variables were reported as percentages and $95 \%$ confidence intervals $(95 \% \mathrm{CI})$. Differences between groups were analyzed with the Chi-square test. A univariate analysis was performed evaluating mITT effectiveness or safety and the presence or absence of statins in the therapy. A multivariate analysis was also carried out using a logistic regression model by means of the stepwise forward likelihood method with $H$. pylori mITT eradication a as dependent variable, and age, gender, treatment duration, PPI doses, compliance and statin's presence/absence, as independent factors. Significance was considered at $p<0.05$.

\section{Conclusions}

In conclusion, our study provides a comprehensive global overview of the inconsistent usefulness of adding statins to eradication therapies against $H$. pylori as a strategy to increase effectiveness. Therefore, the addition of statins to the $H$. pylori treatment cannot be recommended to improve eradication. However, ideally, large randomized studies should be specifically performed to definitively explore this option. Nonetheless, it is important to remark that currently recommended regimens are more complex than those recommended several years ago, showing a tendency to use quadruple instead of triple therapies, advocating for extended duration, and using high PPI doses. The addition of another medication -with the aim of increasing $H$. pylori eradication- has to be carefully weighed as this could affect treatment adherence, which is a key factor affecting the effectiveness.

Supplementary Materials: The following are available online at https:/ /www.mdpi.com/article/ 10.3390/antibiotics10080965/s1. Supplementary file S1: Contribution log. Supplementary file S2: Proton pump inhibitor (PPI) categories: low, standard and high acid inhibition. Supplementary Table S1: Inclusion of patients per country. Supplementary Table S2: Distribution of eradication therapies prescribed according to the statin used. Supplementary Table S3: Adverse events reported in each group according to severity. Supplementary Table S4: Effectiveness and safety in empirical therapies according to the type of statin used. Supplementary Table S5: Effectiveness and safety in culture-guided therapies according to the type of statin used.

Author Contributions: Conceptualization, O.P.N. and J.P.G.; data curation, M.C., Á.P.-A., B.T., A.K.-H., L.B., A.J.L., L.R., D.V., L.F.-S., J.M.H., J.P.-L., N.B.J., G.F., J.B., M.A., J.O., R.P., M.L., J.M.-I., P.B., S.A., M.D.-C., J.G.-C., V.N., S.J.M.-D., R.R.-Z., O.N., A.S.S., P.A., P.P., M.E., I.P., O.P.N., F.M., C.O. and J.P.G.; formal analysis, M.C., O.P.N. and J.P.G.; funding acquisition, J.P.G., methodology, O.P.N. and J.P.G.; project administration, I.P., O.P.N., F.M., C.O. and J.P.G.; resources, J.P.G.; software, O.P.N.; supervision, O.P.N. and J.P.G.; writing—original draft preparation, M.C.; writing-review and editing, Á.P.-A., B.T., A.K.-H., L.B., A.J.L., L.R., D.V., L.F.S., J.M.H., J.P.-L., N.B.J., G.F., J.B., M.A., J.O., R.P., M.L., J.M.-I., P.B., S.A., M.D.-C., J.G.-C., V.N., S.J.M.-D., R.R.-Z., O.N., A.S.S., P.A., P.P., M.E., I.P., O.P.N., F.M., C.O. and J.P.G. All authors have read and agreed to the published version of the manuscript.

Funding: This project was promoted and funded by the European Helicobacter and Microbiota Study Group (EHMSG), the Spanish Association of Gastroenterology (AEG), and the Centro de Investigación Biomédica en Red de Enfermedades Hepáticas y Digestivas (CIBERehd). 
Institutional Review Board Statement: The Hp-EuReg protocol was approved by the Ethics Committee of La Princesa University Hospital (Madrid, Spain), which acted as a reference Institutional Review Board (20 December 2012), and was conducted according to the guidelines of the Declaration of Helsinki, was classified by the Spanish Drug and Health Product Agency, and was prospectively registered at Clinical Trials.gov under the code NCT02328131. An addendum for a ten-year extension of the project was also approved (15 January 2015).

Informed Consent Statement: Informed consent was obtained from all subjects involved in the study.

Data Availability Statement: The data presented in this study are available on request from the corresponding author.

Conflicts of Interest: Ángeles Pérez-Aisa has served as a speaker for Allergan. Luis FernándezSalazar has received honoraria from Norgine, Janssen, and Tillots. Jesús Barrio has served as a speaker, as a consultant, or has received research or education funding from MSD, Abbvie, Takeda, Janssen, and Ferring. Pedro Almela has served as a speaker, a consultant, and an advisory member for or has received research funding from MSD, Abbvie, Takeda, Janssen, Gebro Pharma, Tillotts Pharma, and Biogen. Olga P. Nyssen has received research funding from Allergan. Francis Megraud received honoraria from Phathom (advisory meeting) and Biocodex (lecture fees) and commercial research funding from Aptalis. Javier P. Gisbert has served as speaker, consultant, and advisory member for or has received research funding from Mayoly, Allergan, Diasorin, Gebro Pharma, and Richen. The rest of the authors have no disclosures.

\section{References}

1. Malfertheiner, P.; Megraud, F.; O’Morain, C.A.; Gisbert, J.P.; Kuipers, E.J.; Axon, A.; Bazzoli, F.; Gasbarrini, A.; Atherton, J.; Graham, D.J.; et al. Management of Helicobacter pylori infection-the Maastricht V/Florence Consensus Report. Gut 2017, 66, 6-30. [CrossRef]

2. Crowe, S.E. Helicobacter pylori Infection. N. Engl. J. Med. 2019, 380, 1158-1165. [CrossRef]

3. Pellicano, R.R.D.; Fagoonee, S.; Astegiano, M.; Saracco, G.M.; Mégraud, M. A 2016 panorama of Helicobacter pylori infection: Key messages for clinicians. Panminerva Med. 2016, 58, 304-317.

4. Graham, D.Y.; Lu, H.; Yamaoka, Y. Therapy for Helicobacter pylori infection can be improved: Sequential therapy and beyond. Drugs 2008, 68, 725-736. [CrossRef] [PubMed]

5. Fallone, C.A.; Moss, S.F.; Malfertheiner, P. Reconciliation of Recent Helicobacter pylori Treatment Guidelines in a Time of Increasing Resistance to Antibiotics. Gastroenterology 2019, 157, 44-53. [CrossRef] [PubMed]

6. Megraud, F.; Coenen, S.; Versporten, A.; Kist, M.; Lopez-Brea, M.; Hirschl, A.; Andersen, L.P.; Goossens, H.; Glupczynski, Y. Helicobacter pylori resistance to antibiotics in Europe and its relationship to antibiotic consumption. Gut 2013, 62, 34-42. [CrossRef]

7. Nyssen, O.P.; Bordin, D.; Tepes, B.; Perez-Aisa, A.; Vaira, D.; Caldas, M.; Bujanda, L.; Castro-Fernadez, M.; Lerang, F.; Leja, M.; et al. European Registry on Helicobacter pylori management (Hp-EuReg): Patterns and trends in first-line empirical eradication prescription and outcomes of 5 years and 21,533 patients. Gut 2021, 70, 40-54. [CrossRef] [PubMed]

8. Caldas, M.; Pérez-Aisa, M.; Castro-Fernández, M.; Bujanda, L.; Lucendo, A.J.; Rodrigo, L.; Huguet, J.M.; Pérez-Lasala, J.; Molina-Infante, J.; Barrio, J.; et al. European Registry on Helicobacter pylori Management: Effectiveness of First and Second-Line Treatment in Spain. Antibiotics 2020, 10, 13. [CrossRef] [PubMed]

9. Gisbert, J.P.; McNicholl, A.G. Optimization strategies aimed to increase the efficacy of, H. pylori eradication therapies. Helicobacter 2017, 22, e12392. [CrossRef]

10. Li, C.; Shi, Y.; Suo, B.; Tian, X.; Zhou, L.; Song, Z. PPI-amoxicillin dual therapy four times daily is superior to guidelines recommended regimens in the Helicobacter pylori eradication therapy within Asia: A systematic review and meta-analysis. Helicobacter 2021, e12816. [CrossRef]

11. Georgopoulos, S.; Papastergiou, V. An update on current and advancing pharmacotherapy options for the treatment of, $H$. pylori infection. Expert Opin. Pharmacother. 2020, 22, 729-741. [CrossRef]

12. Grundy, S.M.; Stone, N.J.; Bailey, A.L.; Beam, C.; Birtcher, K.K.; Blumenthal, R.S.; Braun, L.T.; De Ferranti, S.; Faiella-Tommasino, J.; Forman, D.E.; et al. 2018 AHA/ACC/AACVPR/AAPA/ABC/ACPM/ADA/AGS/APhA/ASPC/NLA/PCNA Guideline on the Management of Blood Cholesterol. J. Am. Coll. Cardiol. 2019, 73, e285-e350. [CrossRef]

13. Cholesterol Treatment Trialists' (CTT) Collaboration; Baigent, C.; Blackwell, L.; Emberson, J.; Holland, L.E.; Reith, C.; Bhala, N.; Peto, R.; Barnes, E.H.; Keech, A.; et al. Efficacy and safety of more intensive lowering of LDL cholesterol: A meta-analysis of data from 170,000 participants in 26 randomised trials. Lancet 2010, 376, 1670-1681.

14. Dehnavi, S.; Sohrabi, N.; Sadeghi, M.; Lansberg, P.; Banach, M.; Al-Rasadi, K.; Johnston, T.P.; Sahebkar, A. Statins and autoimmunity: State-of-the-art. Pharmacol. Ther. 2020, 214, 107614. [CrossRef] [PubMed] 
15. Pinal-Fernandez, I.; Casal-Dominguez, M.; Mammen, A.L. Statins: Pros and cons. Med. Clínica 2018, 150, 398-402. [CrossRef]

16. Albert, M.A.; Danielson, E.; Rifai, M.N.; Ridker, P.M. Effect of statin therapy on C-reactive protein levels: The pravastatin inflammation/CRP evaluation (PRINCE): A randomized trial and cohort study. JAMA 2001, 286, 64-70. [CrossRef]

17. Deshpande, A.; Pasupuleti, V.; Rothberg, M.B. Statin Therapy and Mortality from Sepsis: A Meta-analysis of Randomized Trials. Am. J. Med. 2015, 128, 410-417.e1. [CrossRef] [PubMed]

18. Wan, Y.-D.; Sun, T.-W.; Kan, Q.-C.; Guan, F.-X.; Zhang, S.-G. Effect of statin therapy on mortality from infection and sepsis: A meta-analysis of randomized and observational studies. Crit. Care 2014, 18, R71. [CrossRef]

19. Cheung, K.-S.; Chan, E.W.; Wong, A.Y.S.; Chen, L.; Seto, W.-K.; Wong, I.C.K.; Leung, W.K. Statins were associated with a reduced gastric cancer risk in patients with eradicated Helicobacter pylori infection: A territory-wide propensity score matched study. Cancer Epidemiol. Biomark. Prev. 2020, 29, 493-499. [CrossRef] [PubMed]

20. Chiu, H.-F.; Ho, S.-C.; Chang, C.-C.; Wu, T.-N.; Yang, C.-Y. Statins Are Associated With a Reduced Risk of Gastric Cancer: A Population-Based Case-Control Study. Am. J. Gastroenterol. 2011, 106, 2098-2103. [CrossRef]

21. Pantea, M.; Negovan, A.; Voidăzan, S.; Macarie, M.; Mocan, S.; Bataga, S.M. Statins and gastroduodenal endoscopic lesions. Medicine 2018, 97, e13579. [CrossRef]

22. Lin, C.-J.; Liao, W.-C.; Lin, H.-J.; Hsu, Y.-M.; Lin, C.-L.; Chen, Y.-A.; Feng, C.-L.; Chen, A.-J.; Kao, M.-C.; Lai, C.-H.; et al. Statins Attenuate Helicobacter pylori CagA Translocation and Reduce Incidence of Gastric Cancer: In Vitro and Population-Based Case-Control Studies. PLoS ONE 2016, 11, e0146432. [CrossRef]

23. Ribaldone, D.G.; Vernero, M.; Caviglia, G.P. Targeting IL-10, ZO-1 gene expression and IL-6/STAT-3 trans-signalling by a combination of atorvastatin and mesalazine to enhance anti-inflammatory effects and attenuate progression of oxazolone-induced colitis. Fundam. Clin. Pharmacol. 2020, 35, 141-142. [CrossRef]

24. Yamato, M.; Watanabe, T.; Higuchi, K.; Taira, K.; Tanigawa, T.; Shiba, M.; Tominaga, K.; Fujiwara, Y.; Oshitani, N.; Takeuchi, K.; et al. Anti-Inflammatory Effects of Pravastatin on Helicobacter Pylori-Induced Gastritis in Mice. Dig. Dis. Sci. 2007, 52, 2833-2839. [CrossRef] [PubMed]

25. Nseir, W.K.J.; Tatour, I.; Haiek, S.; Samara, M.; Assy, N. Long-Term Statin Therapy Affects the Severity of Chronic Gastritis. Helicobacter 2010, 15, 510-515. [CrossRef]

26. Nseir, W.; Diab, H.; Mahamid, M.; Abu-Elheja, O.; Samara, M.; Abid, A.; Mograbi, J. Randomised clinical trial: Simvastatin as adjuvant therapy improves significantly the Helicobacter pylori eradication rate-A placebo-controlled study. Aliment. Pharmacol. Ther. 2012, 36, 231-238. [CrossRef]

27. Liao, W.-C.; Huang, M.-Z.; Wang, M.L.; Lin, C.-J.; Lu, T.-L.; Lo, H.-R.; Pan, Y.-J.; Sun, T.-C.; Kao, M.-C.; Lin, H.-J.; et al. Statin Decreases Helicobacter pylori Burden in Macrophages by Promoting Autophagy. Front. Cell. Infect. Microbiol. $2017,6,203$. [CrossRef]

28. Lai, C.-H.; Chang, Y.-C.; Du, S.-Y.; Wang, H.-J.; Kuo, C.-H.; Fang, S.-H.; Fu, H.-W.; Lin, H.-H.; Chiang, A.-S.; Wang, W.-C. Cholesterol Depletion Reduces Helicobacter pylori CagA Translocation and CagA-Induced Responses in AGS Cells. Infect. Immun. 2008, 76, 3293-3303. [CrossRef] [PubMed]

29. McNicholl, A.G.; O'Morain, C.A.; Megraud, F.; Gisbert, J.P. Protocol of the European Registry on the management of Helicobacter pylori infection (Hp-EuReg). Helicobacter 2019, 24, e12630. [CrossRef] [PubMed]

30. Huang, J.Q.; Hunt, R.H. Treatment after failure: The problem of "non-responders". Gut 1999, 45, I40-I44. [CrossRef]

31. Molina-Infante, J.; Lucendo, A.J.; Angueira, T.; Rodriguez-Tellez, M.; Perez-Aisa, A.; Balboa, A.; Barrio, J.; Martin-Noguerol, E.; Gomez-Rodriguez, J.; Botargues-Bote, J.M.; et al. Optimised empiric triple and concomitant therapy for Helicobacter pylori eradication in clinical practice: The OPTRICON study. Aliment. Pharmacol. Ther. 2015, 41, 581-589. [CrossRef] [PubMed]

32. Graham, D.Y.; Lu, H.; Dore, M.P. Relative potency of proton-pump inhibitors, Helicobacter pylori therapy cure rates, and meaning of double-dose PPI. Helicobacter 2019, 24, e12554. [CrossRef] [PubMed]

33. McNicholl, A.G.; Bordin, D.S.; Lucendo, A.; Fadeenko, G.; Fernandez, M.C.; Войнован, И.; Zakharova, N.V.; Sarsenbaeva, A.S.; Bujanda, L.; Perez-Aisa, A.; et al. Combination of Bismuth and Standard Triple Therapy Eradicates Helicobacter pylori Infection in More than 90\% of Patients. Clin. Gastroenterol. Hepatol. 2020, 18, 89-98. [CrossRef]

34. Nyssen, O.P.; Perez-Aisa, A.; Castro-Fernandez, M.; Pellicano, R.; Huguet, J.M.; Rodeigo, L.; Ortun, J.; Gomez-Rodriguez, B.J.; Pinto, R.M.; Areia, M.; et al. European Registry on Helicobacter pylori management: Single-capsule bismuth quadruple therapy is effective in real-world clinical practice. United Eur. Gastroenterol. J. 2021, 9, 38-46. [CrossRef] [PubMed]

35. Hassan, A.M.; Shawky, M.A.E.-G.; Mohammed, A.Q.; Haridy, M.A.; Eid, K.A.-E.-A. Simvastatin improves the eradication rate of Helicobacter pylori: Upper Egypt experience. Infect. Drug Resist. 2019, 12, 1529-1534. [CrossRef] [PubMed]

36. McNicholl, A.G.; Gisbert, J.P. Commentary: Comparators in, H. pylori eradication—stating the ethics of statins. Aliment. Pharmacol. Ther. 2012, 36, 400-401. [CrossRef]

37. Parsi, A.; Biuseh, M.; Mohammadi, S.; Hajiani, E.; Hashemi, S.J.; Saeidian, H.; Biuseh, M. Study of probiotic and simvastatin additive effect on Helicobacter pylori eradication rate. Int. J. Gastrointest. Interv. 2019, 8, 160-164. [CrossRef]

38. Sarkeshikian, S.S.; Ghadir, M.R.; Alemi, F.; Jalali, S.M.; Hormati, A.; Mohammadbeigi, A. Atorvastatin in combination with conventional antimicrobial treatment of Helicobacter pylori eradication: A randomized controlled clinical trial. J. Gastroenterol. Hepatol. 2019, 35, 71-75. [CrossRef] 
39. Dormuth, C.R.; Filion, K.; Paterson, J.M.; James, M.T.; Teare, G.F.; Raymond, C.B.; Rahme, E.; Tamim, H.; Lipscombe, L.; for the Canadian Network for Observational Drug Effect Studies (CNODES) Investigators. Higher potency statins and the risk of new diabetes: Multicentre, observational study of administrative databases. BMJ 2014, 348, g3244. [CrossRef]

40. Harris, P.A.; Taylor, R.; Thielke, R.; Gonzalez, N.; Conde, J.G. Research electronic data capture (REDCap)—A metadata-driven methodology and workflow process for providing translational research informatics support. J. Biomed. Inform 2009, 42, 377-381. [CrossRef]

41. McNicholl, A.G.; Gisbert, J.P. Research to the N-Power: The Strengths of Networked Clinical Collaboration in Spain. Am. J. Gastroenterol. 2017, 112, 1761-1764. [CrossRef] [PubMed]

42. Kirchheiner, J.; Glatt, S.; Fuhr, U.; Klotz, U.; Meineke, I.; Seufferlein, T.; Brockmöller, J. Relative potency of proton-pump inhibitors-comparison of effects on intragastric pH. Eur. J. Clin. Pharmacol. 2009, 65, 19-31. [CrossRef] [PubMed] 